University of Nebraska - Lincoln

DigitalCommons@University of Nebraska - Lincoln

U.S. National Park Service Publications and

Papers

National Park Service

2015

State-space modeling to support management of brucellosis in the Yellowstone bison population

\author{
N. Thompson Hobbs \\ Colorado State University, tom.hobbs@colostate.edu \\ Chris Geremia \\ National Park Service \\ John Treanor \\ National Park Service \\ Rick Wallen \\ National Park Service \\ P. J. White \\ National Park Service
}

See next page for additional authors

Follow this and additional works at: https://digitalcommons.unl.edu/natlpark

Thompson Hobbs, N.; Geremia, Chris; Treanor, John; Wallen, Rick; White, P. J.; Hooten, Mevin B.; and Rhyan, Jack C., "State-space modeling to support management of brucellosis in the Yellowstone bison population" (2015). U.S. National Park Service Publications and Papers. 127.

https://digitalcommons.unl.edu/natlpark/127

This Article is brought to you for free and open access by the National Park Service at DigitalCommons@University of Nebraska - Lincoln. It has been accepted for inclusion in U.S. National Park Service Publications and Papers by an authorized administrator of DigitalCommons@University of Nebraska - Lincoln. 


\section{Authors}

N. Thompson Hobbs, Chris Geremia, John Treanor, Rick Wallen, P. J. White, Mevin B. Hooten, and Jack C. Rhyan 


\title{
State-space modeling to support management of brucellosis in the Yellowstone bison population
}

\author{
N. Thompson Hobbs, ${ }^{1,5}$ Chris Geremia, ${ }^{2}$ John Treanor, ${ }^{2}$ Rick Wallen, ${ }^{2}$ P. J. White, ${ }^{2}$ Mevin B. Hooten, ${ }^{3}$ and \\ JACK C. RHYAN ${ }^{4}$ \\ ${ }^{1}$ Natural Resource Ecology Laboratory, Department of Ecosystem Science and Sustainability, \\ and Graduate Degree Program in Ecology, Colorado State University, Fort Collins, Colorado 80523 USA \\ ${ }^{2}$ National Park Service, P.O. Box 168, Mammoth, Wyoming 82190 USA \\ ${ }^{3}$ U.S. Geological Survey, Colorado Cooperative Fish and Wildlife Research Unit, Department of Fish, Wildlife, \\ and Conservation Biology, and Department of Statistics, Colorado State University, Fort Collins, Colorado 80523 USA \\ ${ }^{4}$ U.S. Department of Agriculture, Animal and Plant Health Inspection Service, Veterinary Services, \\ National Wildlife Research Center, Fort Collins, Colorado 80521 USA
}

Abstract. The bison (Bison bison) of the Yellowstone ecosystem, USA, exemplify the difficulty of conserving large mammals that migrate across the boundaries of conservation areas. Bison are infected with brucellosis (Brucella abortus) and their seasonal movements can expose livestock to infection. Yellowstone National Park has embarked on a program of adaptive management of bison, which requires a model that assimilates data to support management decisions. We constructed a Bayesian state-space model to reveal the influence of brucellosis on the Yellowstone bison population. A frequency-dependent model of brucellosis transmission was superior to a density-dependent model in predicting out-of-sample observations of horizontal transmission probability. A mixture model including both transmission mechanisms converged on frequency dependence. Conditional on the frequency-dependent model, brucellosis median transmission rate was $1.87 \mathrm{yr}^{-1}$. The median of the posterior distribution of the basic reproductive ratio $\left(R_{0}\right)$ was 1.75 . Seroprevalence of adult females varied around $60 \%$ over two decades, but only 9.6 of 100 adult females were infectious. Brucellosis depressed recruitment; estimated population growth rate $\lambda$ averaged 1.07 for an infected population and 1.11 for a healthy population. We used five-year forecasting to evaluate the ability of different actions to meet management goals relative to no action. Annually removing 200 seropositive female bison increased by 30 -fold the probability of reducing seroprevalence below $40 \%$ and increased by a factor of 120 the probability of achieving a $50 \%$ reduction in transmission probability relative to no action. Annually vaccinating 200 seronegative animals increased the likelihood of a $50 \%$ reduction in transmission probability by fivefold over no action. However, including uncertainty in the ability to implement management by representing stochastic variation in the number of accessible bison dramatically reduced the probability of achieving goals using interventions relative to no action. Because the width of the posterior predictive distributions of future population states expands rapidly with increases in the forecast horizon, managers must accept high levels of uncertainty. These findings emphasize the necessity of iterative, adaptive management with relatively short-term commitment to action and frequent reevaluation in response to new data and model forecasts. We believe our approach has broad applications.

Key words: adaptive management; basic reproductive ratio; Bayesian state-space models; Bison bison; Brucella abortus; brucellosis; disease transmission; ecological forecasting; Greater Yellowstone Ecosystem, USA; host-parasite dynamics; serology; uncertainty.

\section{INTRODUCTION}

A fundamental challenge facing ecology in the 21 st century is to inform the pressing environmental issues of the day by bringing together data and understanding in a way that is honest about uncertainty. Here, we describe a Bayesian state-space model of disease transmission in the bison population of the Greater

Manuscript received 29 July 2014; revised 9 February 2015; accepted 19 February 2015; final version received 1 May 2015. Corresponding Editor: A. M. Ellison.

${ }^{5}$ E-mail: Tom.Hobbs@colostate.edu
Yellowstone Ecosystem, USA, that assimilates data from multiple sources to evaluate alternatives for adaptive management. Our general approach is applicable to a diverse range of problems in environmental policy and management.

\section{Data assimilation for models of infectious disease}

Understanding host-parasite dynamics has formed an important challenge for population and community ecology since the seminal paper of Anderson and May (1979). A particularly important contribution of ecology to understanding disease transmission came from 
mathematical analysis of systems of differential equations representing mutually exclusive states of health and disease (Mollison 1995). These analyses provided the basis for identifying key epidemiological parameters in closed form, for example, the basic reproductive ratio of a disease, $R_{0}$, the force of infection, and thresholds for establishment (Keeling and Rohani 2008, Diekman et al. 2012). Ecological models of host-parasite interactions have been used to understand the potential consequences of different mechanisms of transmission, historically emphasizing comparisons between frequency and density dependence. However, the theoretical development and mathematical analysis of ecological models of infectious disease have outpaced their fusion with data (LaDeau et al. 2011). Mathematical analysis predominates recent texts on disease modeling (Keeling and Rohani 2008, Diekman et al. 2012) to the exclusion of methods for model-data assimilation.

One of the reasons that the classic models of infectious disease have not been widely fused with data is the difficulty of parameter estimation for continuous time models, which requires application of methods based on stochastic differential equations (Clark 2007). The discrete-time analogs of continuous-time disease models (e.g., Miller et al. 2006, Yee et al. 2011) are more tractable because data taken at discrete intervals can be matched with model predictions. Parameter estimation and analysis of systems of discrete-time equations, also called matrix models, have seen broad application in population ecology (Caswell 1988). Their use to portray disease dynamics has been advocated (Dobson and Foufopoulos 2001, Oli et al. 2006, Allen and van den Driessche 2008, Klepac and Caswell 2011), but matrix population models representing the influence of disease on host dynamics have rarely been fit with data (Cahn et al. 2011, Muths et al. 2011, Perez-Heydrich et al. 2012). Assimilating matrix population models with data on diseases offers a major advance by applying welldeveloped tools in population ecology to questions in disease ecology.

\section{Brucellosis in Yellowstone bison}

Brucellosis, a disease caused by the bacterium Brucella abortus, was discovered in the bison of Yellowstone National Park in 1917. Brucellosis is readily transmitted to bison, elk (Cervus elaphus), and cattle (Bos primigenius) by exposure of susceptible animals to aborted fetuses and exudates from the reproductive tract of an infected mother (Samartino and Enright 1993, Thorne 2008, Olsen and Tatum 2010). Although infection occurs in male and female ruminants of all ages, it appears that pregnant females are the sole source of transmission. Transmission occurs in an annual pulse coinciding with the birthing interval during late spring (Rutberg 1984). Brucellosis appears to be a chronic disease coevolved to coexist with its host such that host survival is not compromised by infection (Adams 2002). Survival of females is not influenced by brucellosis unless they are acutely infected with another bacterial disease (Joly and Messier 2005).

The Yellowstone bison population offers an unusually valuable opportunity to assimilate data with a discretetime model of population dynamics shaped by disease. There is a rich, multi-decadal database that can be used to support estimation of parameters and to understand the effect of brucellosis on the population's growth rate and its demography. Published research studies provide prior information on the influence of brucellosis on vital rates (Fuller et al. 2007b). The ability to evaluate alternative models of disease transmission is enhanced by periodic reductions in animal numbers following removals by park management. The protracted duration of the disease means that states defined by age and disease status can be represented on the same time scale (Klepac and Caswell 2011). A discrete-time matrix model representing brucellosis dynamics avoids potential problems with discretizing a continuous process (Cooch et al. 2012) because disease transmission coincides with an annual birth pulse.

\section{Modeling to support management of the Yellowstone bison}

The role of Yellowstone National Park in the restoration of bison to its historic range illustrates the value of protected areas to species conservation. However, it also illustrates the limitations of conservation achieved by protection alone. Efforts to control brucellosis in the population by test and culling during the 1930s-1950s were eliminated in 1969 when the park adopted a policy of natural regulation, a policy that considered disease as one of several forces, including severe weather and intraspecific competition for forage, that would regulate the population within the boundaries of the park (Cole 1971). During the 1970s and thereafter, the population grew rapidly after culling ceased (Dobson and Meagher 1996, Plumb et al. 2009). Seasonal movements increasingly extended beyond the park boundaries, bringing bison into the vicinity of livestock on privately owned land surrounding the park (Gates et al. 2005, Kilpatrick et al. 2009, Plumb et al. 2009). The proximity of bison to livestock creates risk of meaningful economic harm to human livelihoods (Bidwell 2010). Thus, brucellosis in the Yellowstone bison is an issue in wildlife policy of national importance (Cheville et al. 1998, Olsen 2010). Management actions are needed to mitigate the risk of disease transmission from bison to livestock (Bidwell 2010).

Yellowstone National Park has embarked on a program of adaptive management of the Yellowstone bison. Adaptive management as it was originally formulated requires a model that assimilates past data to support forecasts of the future behavior of the system being managed (Walters 1986). Bayesian state-space models offer a particularly useful framework for supporting management decisions because they can exploit multiple sources of data, account for multiple 
sources of uncertainty, and provide honest forecasts. They provide a coherent framework for continuously updating current knowledge with new information, the hallmark of adaptive management.

\section{Objectives and Outline of Paper}

Here, we report the development of a discrete-time, state-space model of the Yellowstone bison population. Our objectives were to enhance understanding of the role of brucellosis in shaping bison population dynamics and to provide a basis for forecasting the outcomes of alternative management actions. We believe our modeling approach offers a widely applicable framework for informing decisions in wildlife management and conservation.

The remainder of this paper is organized as follows. We begin with a description of the data that have accrued from population monitoring and designed research studies of bison in Yellowstone during the past four decades. We then outline a Bayesian hierarchical model used to estimate parameters and population states, including future states. We use the model to forecast the effects of different alternatives for managing brucellosis to illustrate how models like ours can be used to support decisions and policy. We close by discussing the implications of our findings for managing bison in Yellowstone and for conserving species in protected areas around the world.

\section{DATA}

Six time series of data were used to estimate model states and parameters and to provide a basis for model selection (Table 1, Fig. 1). These data included: (1) aerial counts, (2) aerial classifications of juveniles and adults, (3) ground classification of adult and yearling males, yearling females, and adult females, (4) seroprevalence of juvenile, yearling, and adult females, (5) a capture history of disease status of yearling and adult females, (6) observations of the number of animals annually removed from the population, and (7) the age, sex, and serology of removals. We will describe the data and the methods for obtaining them.

\section{Total counts and sightability}

Aerial counts of the Yellowstone bison population were conducted during 1970-2010 (Fig. 1A). Counts occurred four times a year, generally in February, MayJune, July-August, and November-December. We used summer counts in our analysis because summer aggregations facilitated accurate counting, and because summer counts were accompanied by age classifications, discussed subsequently (section Age and sex composition). During 35 of the 40 years, there were at least two summer counts, one taken during June and the other during July or August. Bison are social animals that live in large, easily located groups, providing confidence that areas sampled include the preponderance of bison in Yellowstone.
The same observer and pilot conducted all counts during 1970-1997. Census methods have been largely consistent over the four decades of observation. During 1970-1997, observers subjectively chose areas to sample based on observations of large groups from the air. During 1998-2001, observers counted bison in census blocks according to a stratified sampling design to identify areas where bison congregate (Hess 2002). Observers targeted these areas for census during 20022010. Replicated summer counts were used to estimate annual standard deviations in counts, which were used to estimate sampling error for the census data.

We corrected for the failure to observe all animals on any given count using a sightability model developed by Hess (2002). This correction was not large because bison tend to congregate in open habitat and are easily observed during the summer census. On average, the observed number of bison was $97 \%$ of the true number (Hess 2002).

\section{Removals and harvest}

We used data on removals that occurred during 19762010 at the west boundary near West Yellowstone and the north boundary near Gardiner, Montana, USA (Fig. 1A). These data were pooled and combined with estimates of the number of animals harvested by licensed hunters. Data on the sex and age composition of a sample of the removals were available for the years 1985-2010.

\section{Age and sex composition}

The number of calves and the total number of individuals in groups of bison were recorded during annual aerial counts (Fig. 1B). Each group was treated as a single observation taken from a sampling distribution of the proportion of juveniles in the population. The mean of that distribution provided the annual estimate of age composition and the standard deviation provided an accompanying estimate of sampling error, as described in Appendix A. During 2000-2010, age and sex composition of bison groups were also observed from the ground by classifying all individuals within groups into four categories: juveniles, yearling females, adult females, and non-juvenile males (Fig. 1C-E). Parameters of the Dirichlet distribution of sex and age classes were estimated annually following procedures described in Appendix A.

\section{Seroprevalence}

Serologic status is a measure of exposure indicated by the presence of antibodies specific to B. abortus antigen. Juvenile and adult female bison were tested for brucellosis exposure during handling in capture facilities located near park boundaries or by lethal removal after exiting the park (Fig. 1F-H). Disease tests occurred in November through April and depended on the timing of movements to park boundaries. Animals were classified as seropositive or seronegative based on a variety of 
TABLE 1. Summary of data sets used to estimate parameters and states and for out-of-sample validation $\left(\mathbf{Y}_{1}, \mathbf{Y}_{2}, \mathbf{Y}_{3}, \mathbf{Y}_{4}\right)$, where $\mathbf{Y}$ is a matrix of multiple counts for multiple years.

\begin{tabular}{lccr}
\hline \hline \multicolumn{1}{c}{ Parameter data } & Data set & $N_{\dagger}^{\dagger}$ & Time span \\
\hline Mean and SD of replicate counts & $\mathbf{Y}_{1}$ & 41 & $1970-2010$ \\
Aerial classification of adults and juveniles & $\mathbf{Y}_{2}$ & 41 & $1970-2010$ \\
Ground classification of age and/or sex classes & $\mathbf{Y}_{3}$ & 11 & $2000-2010$ \\
Serology by age class & $\mathbf{Y}_{4}$ & 19 & $1985-2010$ \\
Number removed & $\mathbf{y}_{\mathrm{r}}$ & 41 & $1970-2010$ \\
Probability of horizontal transmission & $\mathbf{Y}_{\mathrm{mr}}$ & $14 \times 3$ & $1995-2008$
\end{tabular}

Note: Other data sets were used in the process model and for mark-recapture (mr) model selection $\left(\mathbf{Y}_{\mathrm{mr}}\right) ; \mathbf{y}_{\mathrm{r}}$ is a vector of the total number of animals removed over a series of years.

$\dagger$ Number of years with non-missing data.

tests. Prior to 2000, Montana Department of Livestock Diagnostics Laboratory (Bozeman, Montana, USA) veterinarians created a single seropositive or seronegative classification based on results from a panel of
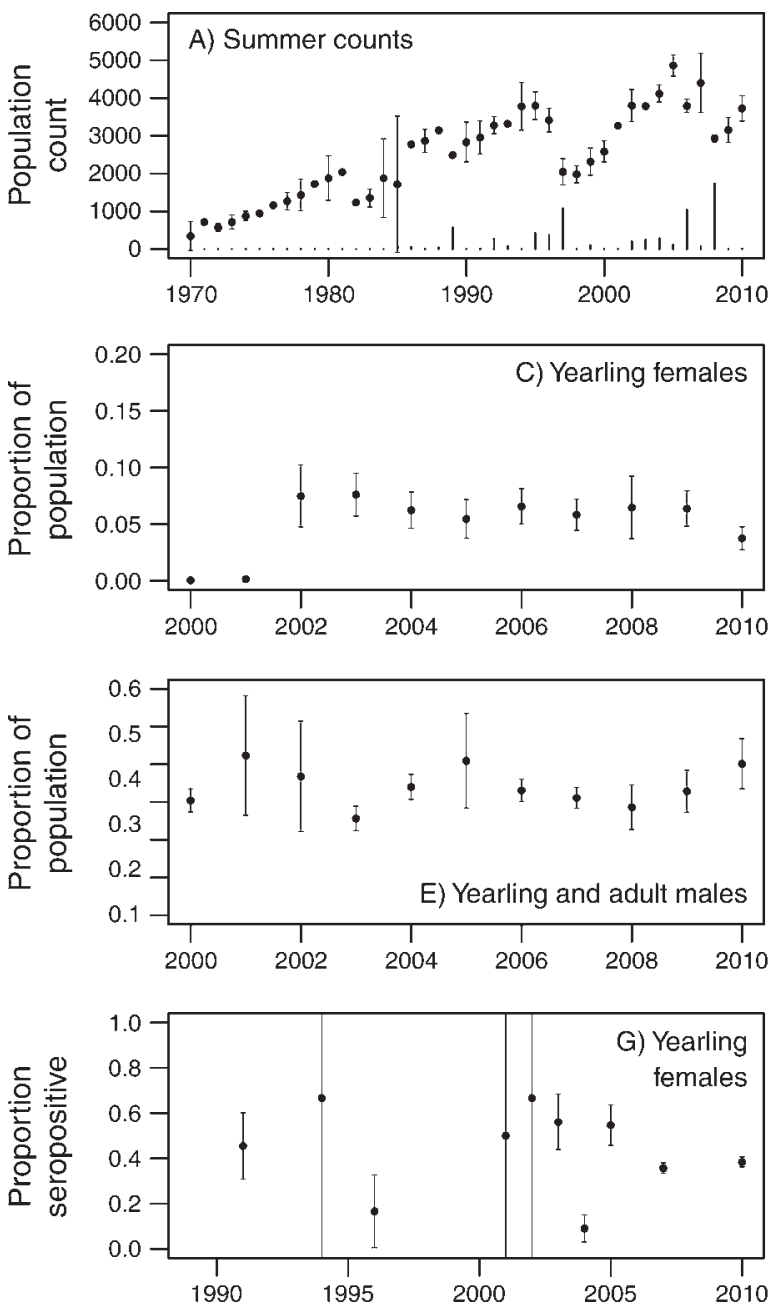

brucellosis serology tests, including at least some of the standard card, buffered-acidified plate antigen, rivanol, complement fixation, particle concentrate fluorescence immunoassay, rapid automated presumptive test, com-
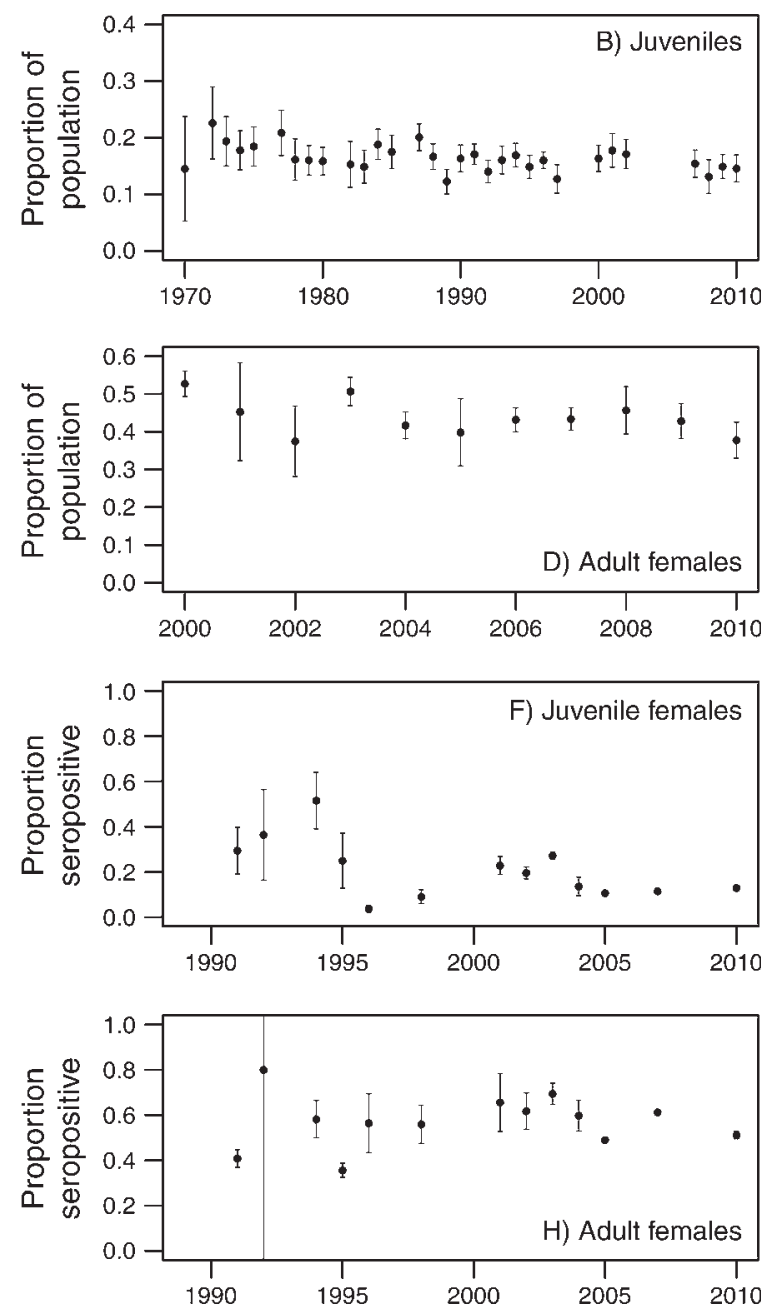

FIG. 1. Time series of data used to estimate states and parameters for Yellowstone bison. Solid circles are means; error bars are \pm 2 standard deviations. (A) Mean of total summer counts across all sex and age classes; vertical lines from the $x$-axis show the total number of animals removed from the population by capture at boundary and hunting. States are shown as the proportion of the population in different age classes: (B) juveniles, (C) yearling females. (D) adult females. (E) Proportion yearling and adult males. The proportion of females testing seropositive for brucellosis is given by age class: $(F)$ juvenile females, $(\mathrm{G})$ yearling females, and (H) adult females. 
petitive enzyme-linked immunoabsorbent assay, standard plate, and standard tube tests (Huber and Nicoletti 1986, Gall et al. 2000, Nielsen and Gall 2001, Nielsen 2002, Philo and Edwards 2002). After 2000, serology status was determined by veterinarians or trained National Park Service biologists using the standard card or fluorescence polarization assay (Gall et al. 2000, Nielsen and Gall 2001). Animal age was recorded at the time of disease testing; e.g., animals classified as juveniles were $\sim 10$ months old and yearlings were 22 months old. Data on seroprevalence for adult females ( $>2$ years old) spanned 1985 to 2010 . Data on juveniles and yearlings were available for 2003 to 2010 .

\section{Infectiousness}

The Animal and Plant Health Inspection Service and the National Park Service conducted a mark-recapture study of adult female bison in Yellowstone National Park and nearby areas of Montana during 1995-2008 to estimate probabilities of transmission of brucellosis. We selected 146 individuals $>1$ year of age (roughly $10 \%$ of the adult female population) using the following procedure. Herds of bison were identified during aerial surveys and their locations were recorded. Subgroups were chosen from herds by choosing a random number $i$ between 1 and the number of subgroups and counting from the front of the herd to the $i$ th subgroup. A second random number $j$ was used to select individuals within subgroups by counting from the front of the subgroup to individual $j$. Animals were added to the study throughout 1995-2008.

Bison were captured in autumn by immobilization with carfentanil and xylazine (Rhyan et al. 2009) or at handling facilities near the boundary of the park (U.S. Department of the Interior National Park Service and U.S. Department of Agriculture Forest Service, Animal, and Plant Health Inspection Service; USDI and USDA 2000). Serologic status was determined using techniques described in the section Seroprevalence. Bison were fitted with a uniquely engraved metal ear tag and mortalitysensing telemetry collar (Advanced Telemetry Systems, Isanti, Minnesota, USA; Lotek, Newmarket, Ontario, Canada; Telonics, Mesa, Arizona, USA) to allow recapture in subsequent years. Using these collars, there is virtually no opportunity for error in the ability to detect when a bison died. We have no records of radio collars not moving into mortality mode when an animal died. Samples for B. abortus culture were collected during 1995-2001, including heparinized blood, milk (if present), feces, and cervical swabs (Rhyan et al. 2009). Isolation of $B$. abortus from samples was conducted at the National Veterinary Services Laboratory according to established standard operating procedures. Attempts were made to catch neonatal calves of associated dams during post-parturition handling (Rhyan et al. 2009). Calves were marked with a unique metal ear tag and recaptured when associated with telemetered dams during the subsequent autumn (Rhyan et al. 2009).
Blood was collected for determining serostatus, and calves were fitted with a telemetry device for long-term monitoring. We observed and/or handled all bison in compliance with the court-negotiated settlement for the Interagency Bison Management Plan (U.S. Department of the Interior, National Park Service and U.S. Department of Agriculture Forest Service, Animal, and Plant Health Inspection Service [USDI and USDA 2000]) and National Park Service research permit YELL-2008-SCI-5340, as well as the guidelines recommended by the American Society of Mammalogists (Sikes et al. 2011). Animal care and welfare procedures were approved by veterinarians from the Biological Resource Management Division of the National Park Service and are described in Wallen and Blanton (2010).

Data were right-censored in response to three events: (1) when a collar malfunctioned, (2) when a study animal died of natural causes or was killed by hunters, or (3) when it was removed from the population by management agencies. When one of these infrequent events occurred, the animal was removed from the database in the previous November to exclude incomplete years of data.

\section{State-Space Model}

We used a fully Bayesian, state-space model to obtain posterior distributions of parameters, latent states, and derived quantities of interest. A model of ecological processes, models linking the processes to data, and models for parameters (Berliner 1996) provided a unified framework for inference:

$$
\begin{aligned}
& {\left[\boldsymbol{\theta}_{\mathrm{p}}, \Theta_{\mathrm{d}}, \mathbf{n}_{(t)}, \mathbf{n}_{(t-1)} \mid \mathbf{Y}_{(1, t)}, \ldots, \mathbf{Y}_{(4, t)}\right]} \\
& \propto \underbrace{\left[\mathbf{n}_{(t)} \mid \boldsymbol{\theta}_{\mathrm{p}}, \mathbf{n}_{(t-1)}\right]}_{\text {process }} \prod_{l=1}^{4} \underbrace{\left[\mathbf{Y}_{(l t)} \mid \boldsymbol{\theta}_{\mathrm{d}(l)}, \mathbf{n}_{(t)}\right]}_{\text {data }}]_{l}^{\left[\boldsymbol{\theta}_{\mathrm{p}}, \Theta_{\mathrm{d}}\right]} .
\end{aligned}
$$

The notation $[a \mid b, c]$ reads the probability or probability density of $a$ conditional on $b$ and $c$. The quantity $\boldsymbol{\theta}_{\mathrm{p}}$ is a vector of parameters in the process model; $\boldsymbol{\theta}_{\mathrm{d}(l)}$ is a vector of parameters in data model $l, \mathbf{n}_{(t)}$ is a vector representing the true, unobserved demographic and disease state of the population at time $t ; \mathbf{Y}$ is a matrix of multiple counts for multiple years. We enclose subscripts that index time, $t$, and data model, $l$, with () to differentiate them from subscripts that are part of the names of a parameter, e.g., $\boldsymbol{\theta}_{\mathrm{d}(l)}$. There are four data models (also called likelihoods), one each for census observations $\left(\mathbf{Y}_{(1 t)}\right)$, aerial classifications $\left(\mathbf{Y}_{(2 t)}\right)$, ground classifications $\left(\mathbf{Y}_{(3 t)}\right)$, and serology $\left(\mathbf{Y}_{(4 t)}\right)$ (Table 1). Thus, $\boldsymbol{\Theta}_{\mathrm{d}}$ is a matrix of parameters in the four data models. It is important to understand that all inference reported in this paper is based on the posterior and joint distributions shown in Eq. 1. We focus on the ecology that stands behind the process model and parameter models in the sections that follow. We present data models in Appendix B and a detailed expression for the posterior and joint distributions in Appendix C. 
TABle 2. The state vector $\mathbf{n}_{(t)}$ is composed of eight age, sex, and disease classes; animals that are infected have been exposed to brucellosis and would return a positive titer, given an accurate test; animals that are infectious are capable of transmitting the disease horizontally and vertically.

\begin{tabular}{|c|c|}
\hline $\begin{array}{l}\text { State } \\
\text { class }\end{array}$ & Definition \\
\hline$n_{1}$ & $\begin{array}{l}\text { Susceptible juveniles (male and female) aged } 0 \text { to } 1 \\
\text { year }\end{array}$ \\
\hline$n_{2}$ & Susceptible yearling females aged $>1$ to 2 years \\
\hline$n_{3}$ & Susceptible adult females aged $>2$ years \\
\hline$n_{4}$ & $\begin{array}{l}\text { Infected, but not infectious, juveniles (male and } \\
\text { female) aged } 0 \text { to } 1 \text { year }\end{array}$ \\
\hline$n_{5}$ & $\begin{array}{l}\text { Infected, but not infectious, yearling females aged }>1 \\
\text { to } 2 \text { years }\end{array}$ \\
\hline$n_{6}$ & Infected and infectious adult females aged $>2$ years \\
\hline$n_{7}$ & $\begin{array}{l}\text { "Recovered" adult females aged }>2 \text { years that can } \\
\text { recrudesce into an infectious state }\end{array}$ \\
\hline$n_{8}$ & Adult and yearling males aged $>1$ year \\
\hline
\end{tabular}

\section{Process model}

We portrayed the dynamics of the Yellowstone bison population using the stochastic model

$$
\log \left(\mathbf{n}_{(t)}\right) \sim \text { multivariate normal }\left(\log \left(A_{(t)} \mathbf{n}_{(t-1)}\right), \sigma_{\mathrm{p}}^{2} \mathbf{I}\right) .
$$

The deterministic model $\mathbf{A}_{(t)} \mathbf{n}_{(t-1)}$ (Oli et al. 2006) predicts the means of the eight element state vector $\mathbf{n}_{(t)}$ (Table 2) based on the true state of the population at $t-$ 1 and parameters in the projection matrix $\mathbf{A}_{(t)}$ (Fig. 2; Appendix D). A single process variance $\sigma_{p}^{2}$ accounts for all the influences on the true state that are not represented by the deterministic model. Thus, the product $\sigma_{\mathrm{p}}^{2}$ with the identity matrix $\mathbf{I}$ is a covariance matrix with $\sigma_{\mathrm{p}}^{2}$ on the diagonal and zeros elsewhere.

We now describe the structure of the deterministic model (Fig. 2) and the biology that stands behind it. The model is not spatially explicit. Although there is evidence that the population is made up of two different herds that spend their summers in the northern and central portions of Yellowstone National Park (Olexa and Gogan 2007), we justify our decision to treat the population without spatial structure as a first approximation of its behavior and because recent evidence suggests that substantial movement between herds occurs annually (Gates et al. 2005, Fuller et al. 2007, White and Wallen 2012). Choices of age and sex classes (Table 2) were guided in part by knowledge of bison life history. We chose two age classes for non-juvenile females because pregnancy and birth rates do not show any effect of age beyond age three years (Fuller et al. 2007) and because it is reasonable to assume that adult survival is high and constant with age in adults (Eberhardt 2002, Pyne et al. 2010). Our choice of classes was also influenced by the data available to fit the model. We used a single age class for juveniles and two age classes for non-juvenile females because sex and age classifications and serology data (Table 1) did not distinguish among ages beyond juvenile, yearling, and adult classes.
Model census refers to the time point coinciding with $t$ in a discrete-time model. Model census (sensu Caswell 1988) occurs in early to mid-June. The birth pulse occurs immediately before census (Gogan et al. 2005, Jones et al. 2010). Horizontal transmission coincides with the birth pulse as a result of contact between susceptible females and residues resulting from live births and abortions of females in the infectious stage. Management removals to reduce the herd occur predominantly in late winter, threequarters of the way through a time step.

We represent two sources of mortality, natural mortality and management removals, which includes hunting. The probability that animals survive all causes of death except removals is $p_{(j)}$ where $j=1$ indexes juveniles, $j=2$ indexes yearling and adult females, and $j$ $=3$ indexes yearling and adult males. The probability that an animal in state $i$ is removed during $t-1$ to $t$ is $r_{(i, t)}$. The probability that an animal survives both sources of mortality during $t-1$ to $t$ is

$$
s_{(i, t)}=p_{\left(\mathbf{a}_{(i)}\right)}-p_{\left(\mathbf{a}_{(i)}\right)}^{(1-q)} r_{(i, t)}
$$

where $q$ is the proportion of the year elapsed before removal, which we treated as a constant (3/4) to correspond to the observed timing of removals. The subscript vector a matches $p_{j}$ to stage $i$. This formulation allows $p$ to be time invariant, while $r_{(i, t)}$ varies annually, informed by data. When $r_{(i, t)}=0$, as occurred during most years, the total mortality is due to natural causes. The derivation of Eq. 3 and details on estimating the random variable $r_{(i, t)}$ from data are given in Appendix E.

We defined horizontal transmission as exposure of susceptible individuals after contact with afterbirth, live calves, and fetuses aborted from infectious females. All exposed animals are infected with the Brucella pathogen and would return a seropositive titer, given an accurate test. However, animals must be reproductive to be infectious, which means that state $n_{6}$ is infectious, whereas states $n_{4}$ and $n_{5}$ are infected but not infectious. Animals can become infectious by exposure as susceptible yearlings and adults, by maturation of subadults exposed earlier in life, or by recrudescence (Fig. 2).

Recovered adult females experiencing an earlier, acute infection could become infectious again via recrudescence that occurs before the birth pulse (Treanor et al. 2010). Animals that have recovered from an acute infection harbor B. abortus at low levels (Treanor et al. 2011). These bacteria can episodically give rise to an active infection in the reproductive tract. Thus, a proportion $(\psi)$ of recovered animals alive during the birth pulse is infectious.

The probability that a susceptible animal becomes infected during a single time step was estimated using a mixture model of disease transmission. The continuoustime, per capita rate of creation of new infections was portrayed as

$$
\frac{1}{S} \times \frac{d S}{d t}=\frac{\beta I}{N^{z}}
$$


Births and transmission to juveniles

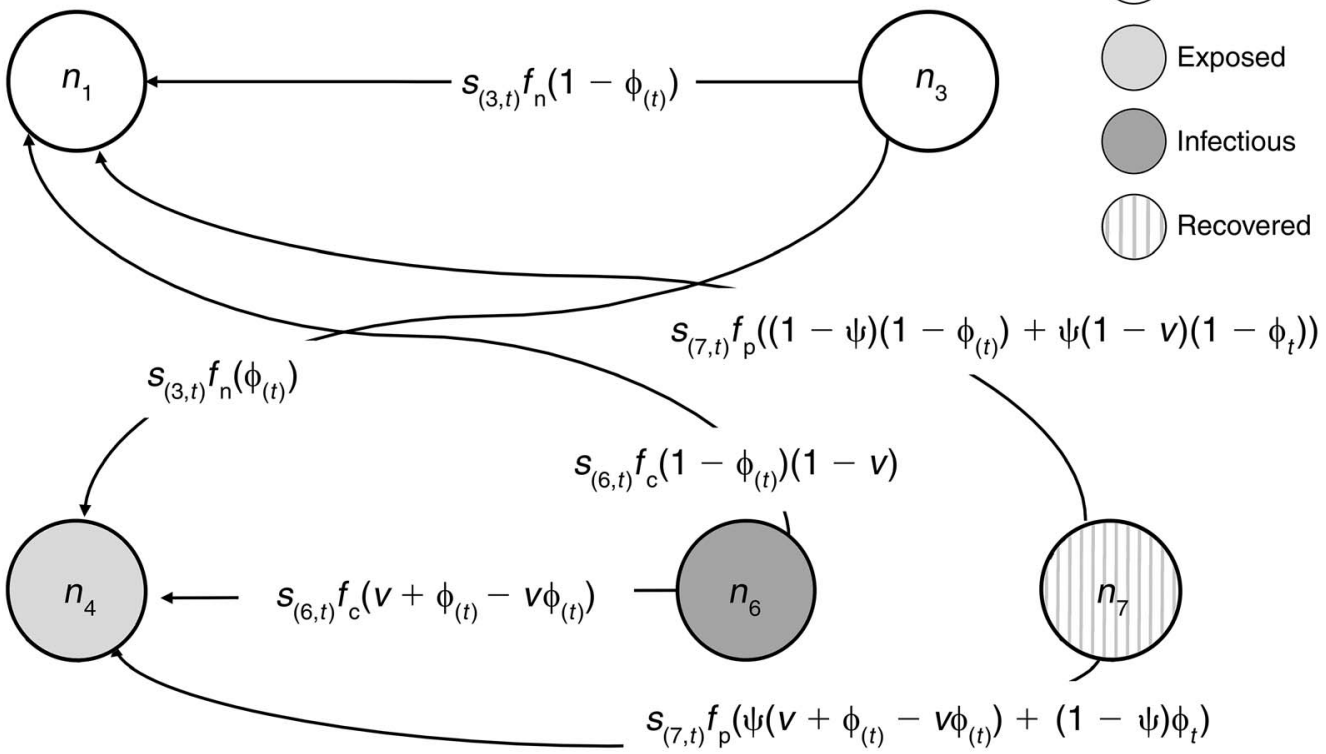

Survival and transmission to yearlings and adults

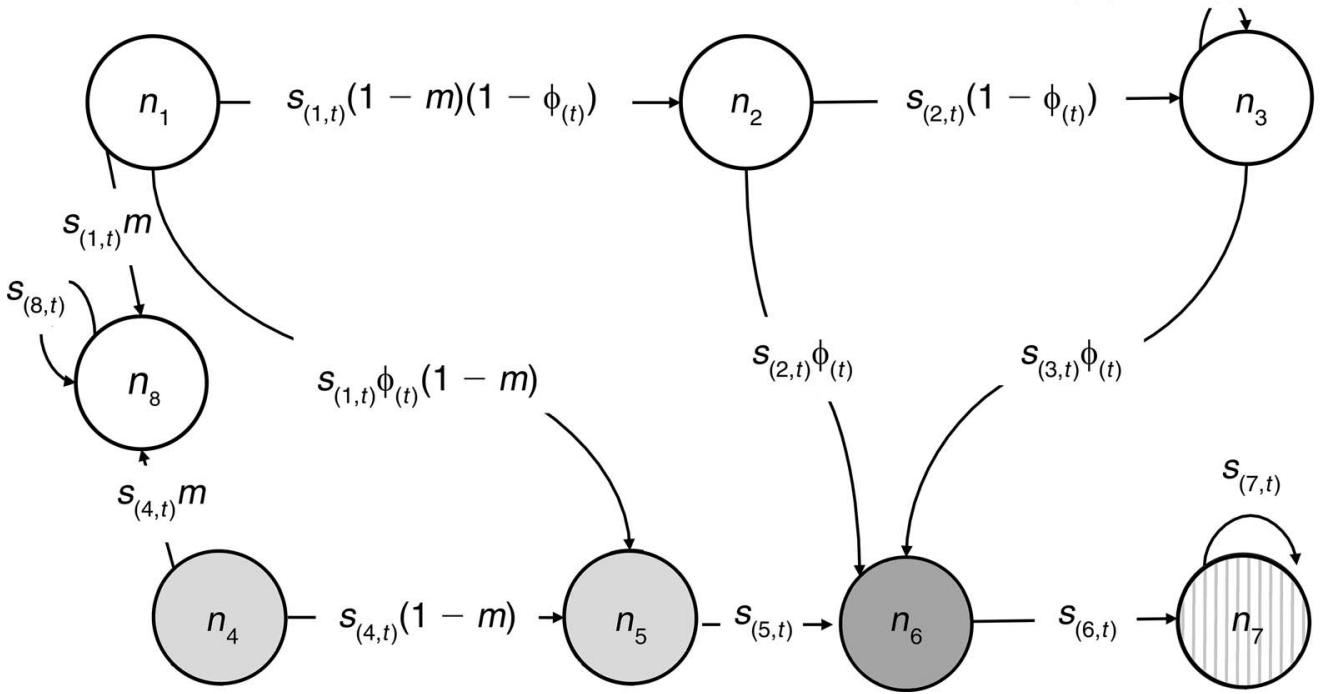

FIG. 2. Model of brucellosis transmission in the Yellowstone bison population. Elements of the state vector $\mathbf{n}_{(t)}$ are defined in Table 2. Parameters are: $f_{\mathrm{n}}$, number of juveniles recruited per seronegative adult female; $f_{\mathrm{p}}$, number of juveniles recruited per seropositive adult female; $f_{\mathrm{c}}$, number of juveniles recruited per seroconverting (infectious) adult female; $\psi$, proportion of recovered population that is infectious; $v$, probability of vertical transmission; $\phi_{(t)}$, probability that a susceptible female becomes exposed during $t-1 \rightarrow t$; and $m$, proportion of juveniles surviving to becomes yearling males. The survival probability $s_{(i, t)}$ reflects natural mortality and management removals 3 . Survival in the absence of removals is $p_{1}$ for juveniles, $p_{2}$ for yearling and adult females, and $p_{3}$ for yearling and adult males. We estimate $\phi_{(t)}$ at each time step using Eq. 4, and $s_{(i, t)}$ using Eq. 3 as described in the section Process model. The dependence of $\phi_{(t)}$ on $\mathbf{n}_{(t)}$ means that the model is nonlinear. See Appendix D for the projection matrix.

where $S$ is the number of susceptibles, $I$ is the number of infecteds, $N$ is the total number of females, $\beta$ is the transmission rate $\left(\mathrm{yr}^{-1}\right)$, and $z$ is a mixing parameter. As the parameter $z$ goes to 1 , the model represents frequency dependence; as $z$ approaches 0 , the model represents density dependence. The probability $\left(\phi_{(t)}\right)$ that a single susceptible becomes infected during $t-1$ to $t$ is $\phi_{(t)}=1-$ 
TABLE 3. Prior distributions for parameters in the model of brucellosis in the Yellowstone bison population, with sources given for informative priors.

\begin{tabular}{|c|c|c|}
\hline Parameter & Definition & Distribution \\
\hline$\beta$ & Rate of transmission $\left(\mathrm{yr}^{-1}\right)$ & uniform $(0,50)$ \\
\hline$f_{\mathrm{n}}$ & Number of offspring recruited per seronegative (susceptible) female & beta $(77,18)$ \\
\hline$f_{\mathrm{p}}$ & Number of offspring recruited per seropositive (recovered) female & beta $(37,20)$ \\
\hline$f_{c}$ & Number of offspring recruited per seroconverting (infectious) adult female & $\operatorname{beta}(3.2,11)$ \\
\hline$m$ & Proportion of juveniles that survive to become yearling males & beta $(49,49)$ \\
\hline$\psi$ & Probability of recrudescence & beta $(2.02,55.6)$ \\
\hline$p_{\text {sight }}$ & Probability of counting an animal that is present in the census area & beta(370,9.5) \\
\hline$\sigma_{\mathrm{p}}$ & Process standard deviation on log scale & uniform $(0,5)$ \\
\hline$p_{1}$ & Probability of survival of juveniles excluding effects $\dagger$ & uniform $(0,1)$ \\
\hline$p_{2}$ & Probability of survival of yearling and adult females & $\operatorname{beta}(40,1.7)$ \\
\hline$p_{3}$ & Probability of survival of yearling and adult males & uniform $(0,1)$ \\
\hline$v$ & Probability of vertical transmission & beta $(1.4,13)$ \\
\hline$\pi$ & Probability of a positive result from a serological test of an animal tha & beta $(476,42)$ \\
\hline$\rho$ & Probability of a positive result from a serological test of an animal that is truly seronegative & beta $(403,15467)$ \\
\hline
\end{tabular}

$\dagger$ Survival probabilities exclude annually varying effects of management removals.

$\exp \left(-\beta I \Delta t / N^{z}\right)$. Converting $I$ and $N$ to the relevant

quantities and assuming $\Delta t=1$ year:

$$
\phi_{(t)}=1-\exp \left(\frac{-\beta\left(s_{(6, t)} n_{(6, t-1)}+\psi s_{(7, t)} n_{(7, t-1)}\right) \Delta t}{\left((1-m) \sum_{i \in\{1,4\}} s_{(i, t)} n_{(i, t-1)}+\sum_{i \in\{2,3,5,6,7\}} s_{(i, t)} n_{(i, t-1)}\right)^{z}}\right) .
$$

The numerator of Eq. 4 includes the number of infectious females that are alive immediately before the birth pulse and the denominator is the total number of females that are alive immediately before the birth pulse. Males are excluded from Eq. 4 because they never become infectious and hence do not participate in the transmission process. The formulation for frequency dependence (i.e., when $z=1$ ) implies that the addition of newborn females to the population at the birth pulse does not meaningfully change the probability of transmission.

We assumed that the number of offspring surviving to census per adult female alive at the previous census (hereafter fecundity, $f$ ) was a function of the mother's brucellosis status, but was not influenced by population density. Earlier empirical work found nominal support for an effect of density on recruitment (Fuller et al. 2007a). We estimated separate, time-invariant fecundities for seronegative animals $\left(f_{\mathrm{n}}\right)$, animals that have seroconverted and are actively infectious $\left(f_{\mathrm{c}}\right)$, and recovered animals that are seropositive $\left(f_{\mathrm{p}}\right)$; see Fig. 2A and Fuller et al. 2007b). These terms include neonatal survival as well as the number of offspring born per female. Recovered animals exhibited higher fecundity than acutely infected animals, owing to some immunoprotection (Treanor et al. 2010). Therefore, the time-invariant fecundity $\left(f_{\mathrm{p}}\right)$ for recovered bison was applied to both recovered and recrudesced individuals. The timing of events in the model means that adult female survival from census to the birth pulse must be included in all expressions for number of offspring annually added to the population (Noon and Sauer 1992); see Fig. 2.
Transmission to young of the year could occur horizontally (Fig. 2A) or vertically from mothers that have active reproductive tract infections (Catlin and Sheehan 1986). Females in the recovered adult stage at time $t$ can recrudesce into the infectious stage before the birth pulse and can produce vertically infected offspring at time $t+1$ with probability $\psi v$. The expression for disease states of offspring produced by the recrudesced class is somewhat complicated (Fig. 2). We explain it in Appendix F.

The model omits covariates describing weather conditions, e.g., drought severity, which have been included in other models of bison population dynamics in Yellowstone (Fuller et al. 2007a). We justify this omission because our central objective was to develop a forecasting model. We we use the term forecast to mean predictions of future states accompanied by coherent estimates of uncertainty arising from the failure of the model to represent all of the influences that shape the population's future trajectory. These influences are rightfully represented in the process variance (Eq. 2), which determines the dispersion of posterior process distributions used to evaluate management actions as described in the section Evaluating alternatives for management.

\section{Parameter models}

We used informative prior distributions for probability of vertical transmission and recrudescence derived from mark-recapture studies (section Infectiousness) and for fecundity parameters reported in Fuller et al. (2007b); see Table 3. The model of Fuller et al. (2007a) for bison fecundity includes the Palmer Drought 
TABLE 3. Extended.

\begin{tabular}{lcl}
\hline \hline Mean & SD & \multicolumn{1}{c}{ Source } \\
\hline 25 & 14.3 & vague \\
0.81 & 0.04 & Fuller et al. (2007b) \\
0.64 & 0.06 & Fuller et al. (2007b) \\
0.22 & 0.10 & Fuller et al. (2007b) \\
0.5 & 0.05 & weakly informative \\
0.035 & 0.024 & this study, section Infectiousness \\
0.97 & 0.01 & Hess (2002) \\
2.5 & 1.4 & vague \\
0.5 & 0.29 & vague \\
0.95 & 0.03 & this study, section Infectiousness \\
0.5 & 0.29 & vague \\
0.09 & 0.07 & this study, section Infectiousness \\
0.92 & 0.011 & Gall et al. (2000), Nielsen and Gall (2001) \\
0.025 & 0.0012 & Gall et al. (2000), Nielsen and Gall (2001) \\
\hline
\end{tabular}

Severity Index (PDSI) as a covariate. We used intercept values from their model, which are fecundities for each disease state at the average PDSI, which by definition is 0 . We obtained parameters for informative beta distributions by moment-matching means and confidence intervals reported in the paper.

Priors on sighting probability were based on Hess (2002). We assumed a weakly informative prior on the sex ratio of juveniles surviving to the yearling age (mean $=0.5, \mathrm{SD}=0.05$ ).

We modeled uncertainty in data on exposure to brucellosis (section Seroprevalence) using a meta-analysis of studies of serological test sensitivity and specificity (Gall et al. 2000, Nielsen and Gall 2001, Gall and Nielsen 2004). We estimated the parameters for the distribution of the probability of a true positive and the probability of a false positive using published values, aggregating results across tests (Table 3 ).

Other prior distributions were chosen to be vague. No prior distribution is completely uninformative. We assured that vague priors did not exert meaningful influence on posteriors by varying choices of prior distributions and their parameters and observing effects of that variation on posteriors (Hobbs and Hooten 2015).

Capture histories (section Infectiousness) were used to estimate parameters of posterior distributions of the probability of vertical transmission and recrudescence, which then served as informative prior distributions in our model (Table 3). Calves associated with dams were captured during October and tested for exposure to brucellosis (section Infectiousness). We assumed that seronegative dams could not expose their calves to $B$. abortus and attributed all exposure of those bison to horizontal routes. Passive antibodies resulting from the transfer of antibodies from seropositive dams to calves are usually undetectable by 5-6 months of age (Rhyan et al. 2009). Calves born to seropositive dams remaining seropositive during October were assumed to be exposed to $B$. abortus through either vertical or horizontal transmission. In these analyses, horizontal $(\phi)$ and vertical $(v)$ transmission were assumed to be time invariant. It follows that

$$
\begin{aligned}
{\left[\phi, v \mid Y_{\mathrm{mr}}\right] \propto } & \prod_{i=1}^{12} \prod_{t \in y_{\operatorname{mr} \text { index }(i)}} \operatorname{Bernoulli}\left(y_{\operatorname{mr}(i, t)} \mid \phi+v-\phi v\right) \\
& \times \prod_{i=1}^{13} \prod_{t \in s_{i}} \operatorname{Bernoulli}\left(y_{\operatorname{mr}(i, t)} \mid \phi\right) \operatorname{beta}(\phi \mid 1,1) \\
& \times \operatorname{beta}(v \mid 1,1)
\end{aligned}
$$

where $\mathbf{Y}_{\mathrm{mr}}$ is the mark-recapture (mr) data set described in section Infectiousness, and $y_{\operatorname{mr} \cdot \operatorname{index}(i)}$ is the subset of years for which serological status was observed for animal $i$. We estimated recrudescence $(\psi)$ using the subset of capture histories that were representative of individuals classified as recovered in our model (section Infectiousness). These animals were determined as seropositive and more than one year had elapsed since seroconverting as a juvenile and reaching reproductive maturity or seroconverting as an adult. Here, $y_{\text {mrc }}$ refers to the number of females where a positive culture result from milk, blood, or vaginal swab was obtained during the subsequent calving period (after the initial brucellosis test) and $n_{\text {mrc }}$ was the total number of females tested. Thus, we estimated the posterior distribution of $\psi$ from mark-recapture studies (section Infectiousness) using

$$
\left[\psi \mid y_{\mathrm{mrc}}, n_{\mathrm{mrc}}\right] \propto \operatorname{beta}\left(y_{\mathrm{mrc}}+1, n_{\mathrm{mrc}}-y_{\mathrm{mrc}}+1\right) .
$$

Dynamic models require estimating initial conditions as a parameter. We estimated the median number of individuals in each age, sex, and disease state during the initial year as

$$
\begin{gathered}
\mathbf{n}_{(1)} \sim \sim \text { init.n } \cdot \operatorname{Dirichlet}(1,1,1,1,1,1,1,1) \\
\text { init.n } \sim \text { gamma }\left(\frac{y_{\mathrm{N} . \text { obs }(1)}^{2}}{y_{\mathrm{N} . \operatorname{var}(1)}}, \frac{y_{\mathrm{N} . \mathrm{obs}(1)}}{y_{\mathrm{N} . \operatorname{var}(1)}}\right)
\end{gathered}
$$

where $y_{\mathrm{N} . o b s(1)}$ is the mean aerial counts at time 1 and $y_{\mathrm{N} \text {.var(1) }}$ is the observed sample variance in the aerial counts at time 1 .

\section{Parameter estimation}

Posterior distributions of parameters and states were estimated using Markov chain Monte Carlo (MCMC) methods implemented in JAGS 3.1.0 (Plummer 2003, 2011a) using the rjags package (Plummer 2011b) of the R computing environment (R Core Team 2012). Three chains were computed for each parameter, with initial values chosen to be diffuse relative to posterior distributions (Brooks and Gelman 1988). Chains were adapted for optimal sampling using 50000 iterations. After discarding the subsequent 50000 iterations as burn-in, we accumulated 100000 samples from each chain. If there was evidence of lack of convergence, additional iterations were added to burn-in and/or retained samples until convergence was achieved. Convergence was assured by 
visual inspection of trace plots to assure stationarity and mixing, and by the diagnostics of Gelman (Gelman and Rubin 1992, Brooks and Gelman 1997) and Heidelberger (Heidelberger and Welch 1983) implemented in the coda package (Plummer et al. 2010) in R. Code for computations is provided in the Supplement.

\section{Model Analysis \\ Posterior predictive checks}

We conducted posterior predictive checks to evaluate the fit of the model to the data (Gelman et al. 2004, Gelman and Hill 2009). Posterior predictive checks use a test statistic $(T)$ calculated from the observed data $\left(T^{\mathrm{obs}}\right)$ and from replicated data sets simulated from the posterior predictive distribution $\left(T^{\mathrm{rep}}\right)$. Bayesian $P$ values, $P_{\mathrm{B}}$, are defined as the probability that the test statistic calculated from the simulated data $\left(y^{\text {rep }}\right)$ is more extreme than the test statistic calculated from the observed data ( $y$ ) (Gelman et al. 2004):

$$
P_{\mathrm{B}}=\operatorname{Pr}\left(T\left(y^{\text {rep }}, \boldsymbol{\theta}\right) \geq T(y, \boldsymbol{\theta} \mid y)\right)=\operatorname{Pr}\left(T^{\mathrm{rep}} \geq T^{\mathrm{obs}}\right)
$$

where $\boldsymbol{\theta}$ is the vector of the parameters in the model. We chose three different test statistics: the mean, standard deviation, and discrepancy statistic. The discrepancy statistic was calculated for the observed and simulated data as

$$
T^{\mathrm{obs}}=\sum_{t=1}^{n}\left(y_{(t)}-\mu_{(t)}\right)^{2}, T^{\mathrm{rep}}=\sum_{t=1}^{n}\left(y_{(t)}^{\mathrm{rep}}-\mu_{(t)}\right)^{2}
$$

where $y_{t}$ is an observation of a state at time $t, y_{t}^{\text {rep }}$ is a simulated value of the state, and $\mu_{t}$ is the model's prediction of the state. We estimated $P_{\mathrm{B}}$ as the proportion of converged iterations for which $T^{\text {rep }}$ exceeded $T^{\mathrm{obs}}$ for the three test statistics. Eq. 8 is a two-tailed probability, which means a model shows lack of fit if $P_{\mathrm{B}}$ is close to 0 or 1 (Gelman et al. 2004). For each iteration in the MCMC algorithm, we simulated a new data set for total population size, proportion of the population in the juvenile class, and age-specific serology. We then calculated the test statistics for each simulated data set and for the observed data and set an indicator variable to 1 if Eq. 8 was true and 0 otherwise. The sum of the indicator variable across all converged iterations divided by the total number of converged iterations was used to approximate $P_{\mathrm{B}}$ for each test statistic and each data set.

\section{Model selection}

We avoided comparisons of large numbers of alternative models, choosing instead to use a lean candidate set focusing on plausible mechanisms of brucellosis transmission. A small, focused set of candidates prevented dilution of inference over many models attempting to reveal manyfold influences on population dynamics. There were three candidate models: frequency dependent where $z=1$ in Eq. 4, density dependent where $z=0$ in Eq. 4, and a combined model where $z$ is an estimated parameter with support over the inclusive interval $0-1$.

We used three metrics to select among the competing models of transmission (Hooten and Hobbs 2015). We withheld data on probability of horizontal transmission obtained in capture-mark-recapture studies (section Infectiousness) from model fitting to allow these data to be used as a basis for model selection using out-ofsample validation. We calculated the mean square prediction error for the probability of horizontal transmission $\left(\mathrm{MSPE}_{\phi}\right)$ for each model as

$$
\operatorname{MSPE}_{\phi}=\sum_{t \in y_{\mathrm{imr}}} \frac{\left(y_{\phi(t)}-\phi_{(t)}\right)^{2}}{14 \sigma_{y_{\phi(t)}}^{2}}
$$

where $y_{\phi(t)}$ is the out-of-sample estimate of the mean transmission probability at time $t ; \sigma_{y_{\phi(t)}}^{2}$ is the variance of the estimate; $y_{\mathrm{imr}}$ is the set of years estimates were made $(n=14)$. We also used the mean of the log predictive density (Gelman et al. 2014) of the probability of transmission

$$
\mathrm{LPPD}_{\phi}=\log \int\left[y_{\phi(t)} \mid \mathbf{Y}, \theta\right][\boldsymbol{\theta} \mid \mathbf{Y}] d \boldsymbol{\theta}
$$

where $\mathbf{Y}$ are the data and $\boldsymbol{\theta}$ is the vector of model parameters. We approximated $\mathrm{LPPD}_{\phi}$ using

$$
\begin{aligned}
& \operatorname{LPPD}_{\phi} \\
& \approx \log \left(\frac{\sum_{k=1}^{K}\left(\prod_{t \in y_{\mathrm{imr}}} \operatorname{beta}\left(y_{\phi(t)} \mid g\left(\phi_{(t)}^{(k)}, \sigma_{y_{\phi(t)}}^{2}\right)\right)\right)}{K}\right)
\end{aligned}
$$

where $k$ indexes iterations in the MCMC algorithm, $K$ is the total number of iterations, and $g()$ is the function that returns the parameters of the beta distribution from arguments mean and variance (Hooten and Hobbs 2015).

The value of the mixing exponent $z$ in the transmission model (Eq. 4) also allowed inference on the relative support in the data for the frequency- and densitydependent models.

\section{Computation of derived quantities}

The equivariance property of the Markov chain Monte Carlo algorithm allows computation of the posterior distribution of quantities derived from parameters and latent variables because the output of any function of a random variable is itself a random variable (Hobbs and Hooten 2015). We computed posterior distributions of derived quantities of interest by drawing values of latent states by sampling from the converged MCMC chains and applying the appropriate function to those values.

A primary objective of our work was to estimate the basic reproductive ratio, $R_{0}$, for brucellosis in the Yellowstone bison. To do so, we used a modification 
of the method described by Allen and van den Driessche (2008) and Oli et al. (2006) for computing $R_{0}$ from the matrix A. Estimating the reproductive ratio of the disease requires estimating the duration of the infectious period and the number of infections produced per unit duration. Details of procedures for estimating $R_{0}$ are provided in Appendix G.

We calculated the effective reproductive ratio of brucellosis $\left(R_{\mathrm{e}(t)}\right)$, the number of new infections at $t$ created per infectious individual alive at time $t-1$ using

$$
R_{\mathrm{e}(t)}=\frac{(1-m) n_{(4, t)}+\phi_{(t)} \sum_{i=1}^{3} s[i, t] n_{(i, t-1)}}{n_{(6, t-1)}+\psi n_{(7, t-1)}} .
$$

We excluded males from the calculation of $R_{\mathrm{e}(t)}$ because they cannot become infectious.

We estimated the annual rate of increase $(\lambda)$ for a bison population infected with brucellosis and for a brucellosis-free population. The transition matrix A was modified to portray a females-only population by setting the parameter $m$ to 0 and by halving the recruitment rates under the assumption of no sex bias in fertility or neonatal survival. Values for $\phi_{(t)}$ were made time invariant by averaging their values over 1975-2010, thereby linearizing A. We modified the matrix A for the healthy population by setting all transmission parameters $(\phi, v, \psi)$ to 0 , and sampling the recruitment rate for all adult female stages from the distribution of the estimate for a seronegative adult female $\left(f_{\mathrm{n}}\right)$.

We estimated the posterior distribution of $\lambda$ by randomly drawing parameter vectors, with replacement, from the output of the MCMC procedure described in section Parameter estimation. We used the eigen function in $\mathrm{R}$ to calculate the dominant eigenvalue of A from the sampled parameter values, thereby obtaining a single value of $\lambda$ for each draw. An accumulation of 50000 of these values was used to describe the probability distribution of $\lambda$ for the healthy and infected populations and the probability distribution of the difference between them. We estimated the posterior distributions of the stable age distribution for a healthy and an infected population including both sexes, using the same sampling procedure.

\section{Evaluating Alternatives for Management}

We evaluated alternatives for management using the following procedure. Our purpose was not to exhaustively compare all alternatives, but rather to illustrate an approach that we believe can be broadly applicable to population and disease management in many different systems.

The ability of different management actions to meet goals for the population was evaluated (Fig. 3) using the predictive process distribution (Hobbs and Hooten 2015) to forecast the state of the population at some specified time in the future. For example, the state might be the total population size, the seroprevalence of brucellosis, or the ratio of adult females to adult males.
A) Do nothing

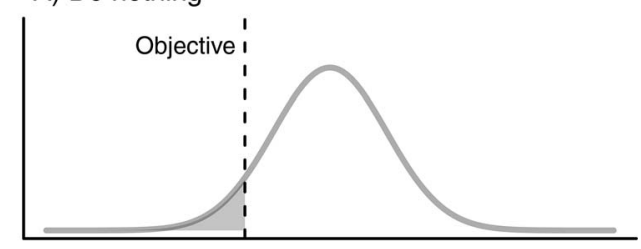

B) Implement management

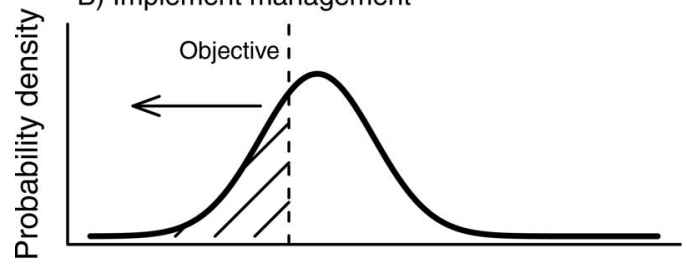

C) Net effect of management

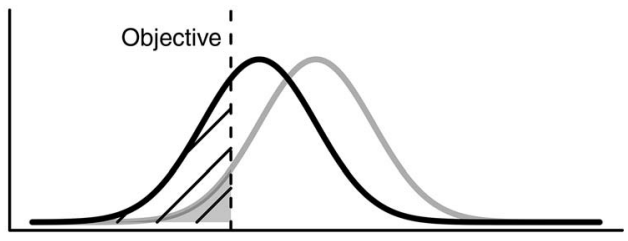

State of population

FIg. 3. We evaluated the effects of alternatives for management using the posterior process distribution of population states. Each distribution $(\mathrm{A}-\mathrm{C})$ is a forecast of the true state of the population at a specified time in the future. For example, that state might be brucellosis prevalence, the total size of the population, or the ratio of adult males to adult females. The gray distributions show the forecast of the future state given no management, and the black distributions show the state forecast if management is implemented. In this example, we seek to reduce the state below the target shown by the vertical dashed line, e.g., the target for an acceptable level of prevalence or probability of infection specified by management. The shaded area in panel (A) gives the probability that the objective will be met at a specified future point in time without taking any action. The effect of a management action is to move the distribution to the left such that the probability of achieving the management target is expanded, as shown by the hatched area in (B). The net effect of management is the ratio of the probability of success when management is implemented, divided by the probability of success with no management. This ratio is the hatched area in $(\mathrm{C})$ divided by the shaded area in (C). A management target specifying that the population should be within a range of values or should exceed an upper threshold can be evaluated using the same approach.

The procedure depends on identifying a desired goal for the state of the population illustrated by the vertical dashed line in Fig. 3. The goal specifies a desired target for the state. For example, the goal might be to reduce seroprevalence below target or to maintain the total population size between an upper and lower target.

Evaluation of alternatives proceeded in three steps. We first obtained the posterior process distribution of the state at some point in the future, given no action, and calculated the probability that the goal will be met (Fig. 3A). The no-action alternative can be considered a null model to which alternative actions can be com- 
pared. Next, we approximated the posterior process distribution at the same point in the future assuming that we have implemented an alternative for management and calculated the probability that the goal will be met (Fig. 3B). Finally, we calculated the ratio of the probability of meeting our goal by taking action over the probability if we take no action. This ratio quantifies the net effect of management (Fig. 3C) and permits statements such as "Taking the proposed action is five times more likely to reduce seroprevalence below $40 \%$ relative to taking no action."

This process for evaluating alternative actions explicitly incorporates uncertainties in the future state of the population in the presence and absence of management. A useful feature of this approach is that the weight of evidence for taking action diminishes as the uncertainty in forecasts increases. That is, increasing uncertainty in forecasts compresses the hatched area in Fig. 3C. This result encourages caution in taking action. Also useful is the inverse relationship between the absolute probability that a goal will be met by management and the probability that it will be met relative to taking no action. As the ambition of objectives increases (e.g., the dashed line in Fig. 3 moves to the left), the absolute probability that the management action will be achieved declines (the hatched area in Fig. 3B shrinks), but the probability of success relative to taking no action increases (the hatched area in Fig. 3C expands). This feature represents a fundamental trade-off in choosing goals and actions that are present in all management decisions: objectives that are not ambitious are easy to meet by applying management, but they might be met almost as easily by taking no action.

Managers in Yellowstone specified four goals. The first was to annually reduce the probability of transmission of brucellosis to half of its current value. The second was to reduce seroprevalence in adult females below $40 \%$. The third was to maintain the total number of bison in the population within 3000-3500 animals, the approximate current population size. Finally, managers sought to maintain the sex and age distribution of the population within the range of values that could be reasonably expected for an exponentially growing population with a stable age distribution.

We evaluated the probability of obtaining these outcomes under five alternatives for management, one to five years into the future, with no uncertainty in the ability of managers to implement each alternative. The first alternative was "no action." We compared four "take action" alternatives for management to the "no action" alternative. We assumed that each of the take action alternatives would target 200 animals annually for five years into the future; that is, five years following the last year that data were taken. The target of 200 was chosen somewhat arbitrarily to allow a comparison among scenarios; we recognize that this target might not be realistically achievable in all scenarios for all years and we deal with that uncertainty below.

In the first "take action" alternative, we assumed that 200 adult and yearling seropositive females would be annually removed. In the second alternative, we assumed that these removals would target seronegative animals. In alternative three, we assumed that 200 animals would be harvested at the boundary. The number of individuals harvested from each population stage class was assumed to be proportional to its abundance in the population.

The fourth "take action" alternative was vaccination, which we implemented by adding two stages to the model (Fig. 4). Stage $n_{(9, t)}$ included susceptible animals that were vaccinated. Stage $n_{(10, t)}$ represented seropositive animals that were exposed to brucellosis after vaccination. This formulation assumes that animals that are vaccinated can become infectious, but are less likely to abort and transmit infection to susceptible animals (Fig. 4). We assumed that vaccination affects the probability of horizontal transmission and vertical transmission in a similar way. We included uncertainty in the efficacy of vaccination by including an informative prior for the parameter $\varepsilon$ (Table 3 ) that represented the proportion of vaccinated animals that were able to transmit the disease vertically and horizontally; thus, $1-$ $\varepsilon$ is the efficacy of the vaccine. We calculated the probability of horizontal transmission as in Eq. 12.

We estimated the distribution of $\varepsilon$ using a betabinomial conjugate prior as $\varepsilon \sim \operatorname{beta}(25+1,74-25+1)$ (Olsen and Johnson 2011; S. C. Olsen, unpublished data), which corresponds to a $56-75 \%$ chance $(95 \%$ BCI, Bayesian credible interval) that a vaccinated animal was protected against transmitting B. abortus. This formulation includes the assumption that vaccinated animals will not be removed from the population.

In these forecasts, we assumed no uncertainty in the ability of managers to annually remove, hunt, or treat 200 animals of the specified brucellosis status. This allowed meaningful comparisons of the ability of each alternative to achieve goals without distorting the comparisons by differences in the number of animals treated under each alternative. However, in reality, there is substantial uncertainty in the number of animals that could realistically be accessed each year. This is because animals must leave the park to be hunted, removed, or vaccinated. The number leaving the park, in turn, is

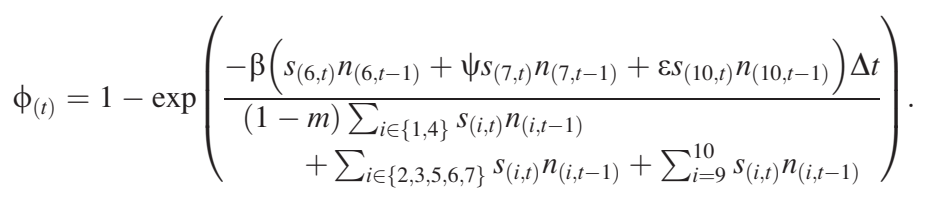



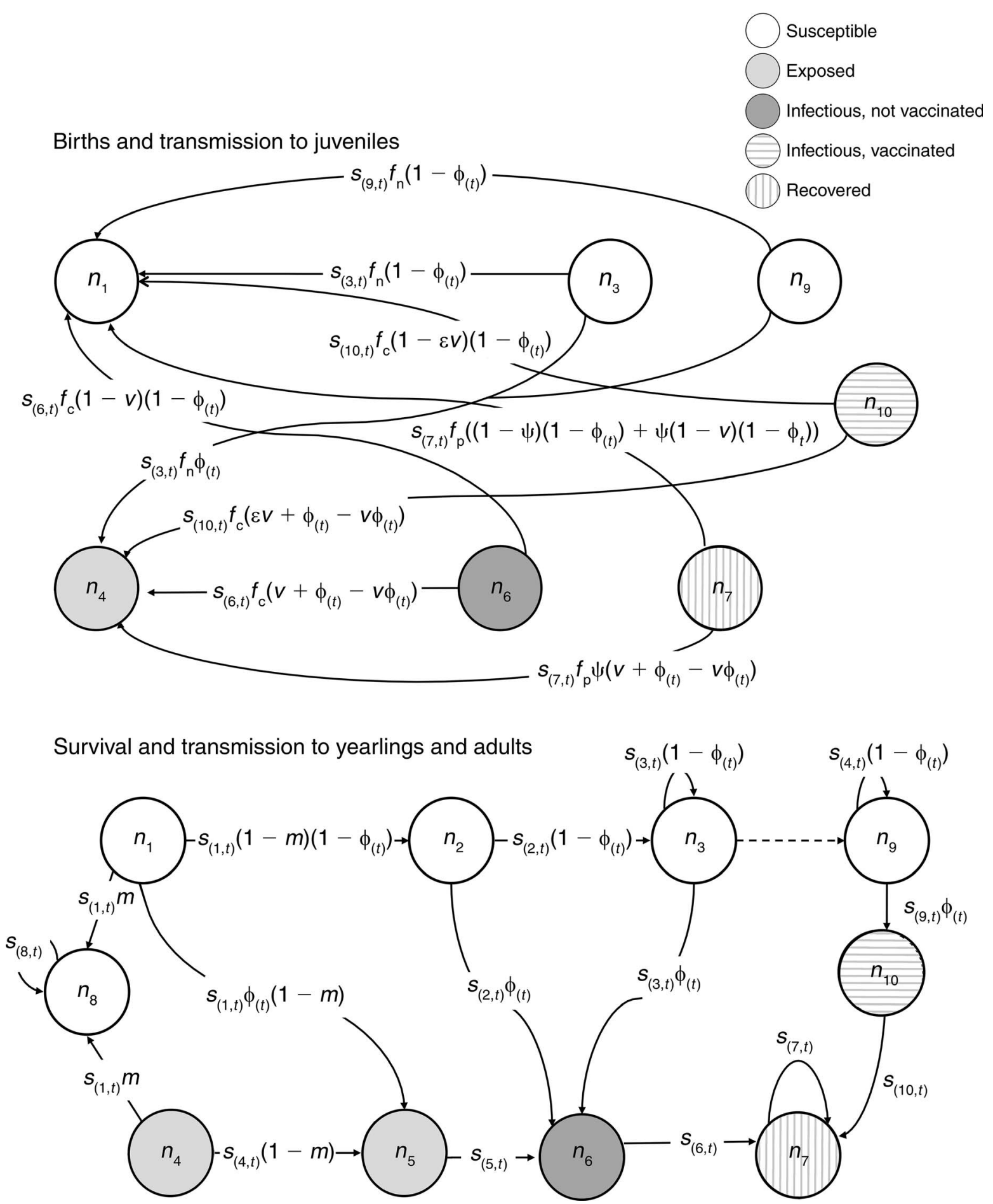

FIG. 4. We added two stages to the model to represent effects of vaccination for brucellosis in the Yellowstone bison population. Stage $n_{(9, t)}$ represents seronegative adult females that have been vaccinated. Stage $n_{(10, t)}$ represents vaccinated adult females that have been exposed. The dashed line represents animals that are vaccinated during a time step. Other stages are defined in Table 2 and parameters are defined in Table 3. The vaccine reduces the probability that exposed individuals can transmit an infection horizontally or vertically, but it does not reduce the probability that an animal can become infected. The random variable $\varepsilon$ represents the proportional reduction in probability of vertical and horizontal transmission that results from vaccination. The survival vector (s) allows annual removals to be included in the model (Eq. 3); however, these were set at zero for the vaccination scenario. 
TABle 4. Bayesian $P$ values for lack of fit between data simulated from posterior predictive distributions and observations for five data sets.

\begin{tabular}{llll}
\hline \hline Model and data set & Discrepancy & Mean & SD \\
\hline Frequency dependent & & & \\
Total population size & 0.51 & 0.5 & 0.51 \\
Proportion juvenile & 0.57 & 0.64 & 0.93 \\
Juvenile serology & 0.8 & 0.75 & 0.81 \\
Yearling serology & 0.13 & 0.058 & 0.19 \\
Adult serology & 0.59 & 0.69 & 0.54 \\
Density dependent & & & \\
Total population size & 0.51 & 0.5 & 0.48 \\
Proportion juvenile & 0.57 & 0.69 & 0.95 \\
Juvenile serology & 0.88 & 0.84 & 0.88 \\
Yearling serology & 0.19 & 0.084 & 0.28 \\
Adult serology & 0.59 & 0.64 & 0.56 \\
Combined & & & \\
Total population size & 0.51 & 0.5 & 0.51 \\
Proportion juvenile & 0.57 & 0.64 & 0.93 \\
Juvenile serology & 0.8 & 0.75 & 0.81 \\
Yearling serology & 0.13 & 0.058 & 0.19 \\
Adult serology & 0.59 & 0.69 & 0.54 \\
\hline
\end{tabular}

Notes: Bayesian $P$ values, $P_{\mathrm{B}}$, are defined as the probability that the test statistic calculated from simulated data is more extreme than the test statistic calculated from observed data. Lack of fit is indicated by values near 1 or 0 . Test statistics were the mean of observations and simulated data, the standard deviation, and the discrepancy, calculated as $\sum_{i=1}^{n}\left(y_{i}-\mu_{i}\right)^{2}$ where $y_{i}$ is an observation, $\mu_{i}$ is the model prediction of the observation, and $n$ is the number of observations in the data set.

influenced by annual variation in winter weather, population size, and forage supplies (Geremia et al. 2011). To incorporate uncertainty in the number of animals that could be accessed for a given alternative (hereafter management uncertainty), we used data on the number of animals removed and the total population size during 1985-2010 to estimate the parameters of a beta distribution representing stochastic variation in the proportion of the population that was removed. We made draws from this beta distribution to estimate the number of animals that were accessible at the boundary $\left(a_{t}\right)$ at future time $t$ as

$$
\begin{aligned}
& \kappa \sim \operatorname{beta}(0.393,4.08) \\
& b_{t}=\kappa \sum_{i=1}^{\text {n.state }} n_{(i, t)}
\end{aligned}
$$

where $n$. state $=10$ for the vaccination alternative and eight for all others. The same value of $b_{t}$ was applied to all management alternatives. The number of animals removed from a given stage was estimated as the product of the total number of animals that were accessible multiplied by the proportion of the stage in the population. So, for example, if the goal was to remove seropositive females, the number removed at time $t$ was calculated as

$$
b_{t} \frac{\sum_{i=5}^{7} n_{(i, t)}}{\sum_{i=1}^{8} n_{(i, t)}} .
$$

It follows that different management alternatives will have different numbers of animals that can be treated in a given year because the animals targeted for treatment represent a different proportion of the total number that could be accessed at the boundary.

We evaluated the probability that goals would be met under each scenario by applying the empirical cumulative distribution function in the $\mathrm{R}$ computing environment (version 2.15.2 R Core Team 2012) to MCMC chains, thereby estimating the areas under the posterior process distribution relative to targets (Fig. 3). To estimate the probability of achieving goals for sex and age composition, we calculated the probability that the model's estimate of the proportion of animals in each sex and age class fell within the $2.5 \%$ and $97.5 \%$ quantiles of the estimated stable age distribution for that class. These quantiles were estimated by sampling from the MCMC chain for each parameter of $\mathbf{A}$, assuming a brucellosis-free population. The posterior distribution of the stable age distribution of $\mathbf{A}$ was then calculated for each draw of parameters using the popbio package (Stubben and Milligan 2007) in R. We estimated the probability of meeting goals for total population size by estimating the area of the posterior predictive distribution of the total population size above 3000 and below 3500; for seroprevalence by estimating the area under its posterior predictive distribution $<0.40$; and for probability of horizontal transmission by estimating the area of the posterior predictive distribution for year five that was less than half the median probability of horizontal transmission in year 0 , the final year that the model was fit to data.

\section{Results \\ Model evaluation and selection}

All diagnostics indicated convergence for the frequency- and density-dependent models with 200000 iterations of three chains following a 50000 iteration burn-in. Trace plots displayed thorough mixing. The $97.5 \%$ quantiles of the Gelman diagnostic was $\leq 1.01$ for all parameters for both models. Merged chains passed the Heidelberger test for stationarity and mean half width. Autocorrelation was less than 0.5 at lag 10 for all parameters except $\sigma_{\mathrm{p}}$. Convergence for the combined model was difficult to achieve. Examination of trace plots showed excellent mixing for all parameters, but autocorrelation was greater than 0.7 at lag 100 for parameters $z$ and $\beta$. All parameters had a $97.5 \%$ quantile $\leq 1.0$ for the Gelman diagnostic after a one million iteration burn-in followed by a 200000 iteration of the three chains.

Model convergence depended on informative prior distributions for fecundity, probability of vertical transmission, and probability of recrudescence. The model would not converge if these distributions were vague.

Posterior predictive checks revealed little evidence of lack of fit between model estimates and data for five 
TABLE 5. Model selection statistics included mean square prediction error $\left(\mathrm{MSPE}_{\phi}\right)$ and deviance of model predictions $\left(\mathrm{LPPD}_{\phi}\right)$ of the probability of infection estimated from outof-sample data from capture-mark-recapture studies.

\begin{tabular}{lccc}
\hline \hline Model & Deviance & $\mathrm{MSPE}_{\phi}$ & $\mathrm{LPPD}_{\phi}$ \\
\hline Frequency & 1359 & 0.77 & 7.62 \\
Combined & 1359 & 0.775 & 7.69 \\
Density & 1360 & 0.901 & 7.52 \\
\hline
\end{tabular}

Notes: Lower values of $\mathrm{MSPE}_{\phi}$ and higher values of $\mathrm{LPPD}_{\phi}$ indicate greater predictive ability. Calculations of model selection statistics are described in Model selection.

data sets (Table 4). Bayesian $P$ values were between 0.12 and 0.88 for 14 out of 15 test statistics for each of the three models. There was some evidence of poor fit of data simulated from the model to observations of the mean of yearling serology for all three models.

The ability of the frequency-dependent and combined models to predict out-of-sample observations of probability of transmission exceeded the predictive ability of the density-dependent model (Table 5), indicating superior ability of models incorporating frequency dependence to predict disease transmission. Estimates of the parameter $z$ in the combined model (Eq. 4) approached 1 (median of posterior distribution of $z=$ 0.914), which means that the combined model was essentially the same as the frequency-dependent form, explaining their similar $\mathrm{MSPE}_{\phi}$ and $\mathrm{PPD}_{\phi}$ values (Table 5). Means of posterior distributions of parameters in the combined and frequency-dependent model were virtually identical for all parameters except $\beta$. We eliminated the density-dependent model from inference because it was less able to predict the probability of transmission relative to the other models and because the estimate of the parameter $z$ ruled out a strong role for density dependence. We eliminated the combined model as a basis for inference because the combined model and frequency-dependent model were mathematically identical when $z=1$ and because convergence required far more iterations relative to the frequency-dependent model. The value of $z$ and the comparisons of parameter estimates between the frequency-dependent and combined model also suggested that inference from a modelaveraged approach would not differ meaningfully from the inferences provided by the frequency-dependent model alone. Consequently, we based all inferences on the frequency-dependent model. Our choice to use a single model means that the inferences we offer are conditional on the model we analyzed.

Comparison of out-of-sample data with model predictions of probability of infection provided confidence that the frequency-dependent model could accurately represent the disease process. Credible intervals on the time-invariant, mark-recapture estimate of probability of horizontal transmission averaged across years overlapped the annual, median model estimate $\left(\phi_{(t)}\right)$ for all years (Fig. 5). Credible limits on model estimatess were within two standard deviations of the

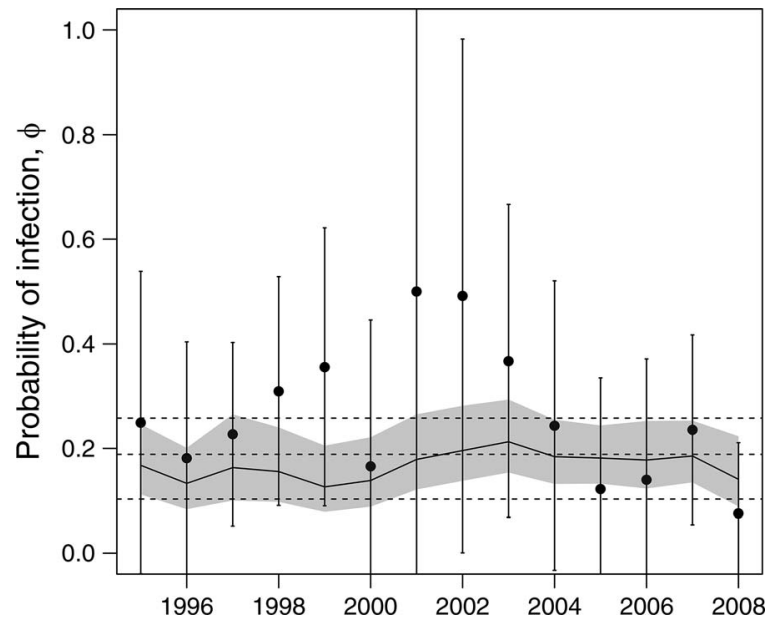

FIG. 5. Median of the posterior distribution (solid line) of the probability that an individual, susceptible bison becomes infected during one year, for the period 1995-2008. The shaded area gives $95 \%$ equal-tailed credible intervals. Points are out-ofsample estimates of infection probability derived from capturemark-recapture studies (section Data: Infectiousness) that were not used to fit the model. Vertical bars are \pm 2 SD of the markrecapture estimate. Horizontal dashed lines are median, 0.025, and 0.975 quantiles of the posterior distribution of the timeinvariant probability of horizontal transmission, based on outof-sample data pooled across all years.

mean of all the time-varying, mark-recapture estimates of infection probability. Credible intervals on model estimates overlapped the annual mean estimates from capture-mark-recapture during all years. The model estimate of the mean proportion of seropositive adult females that were infectious was $0.35(\mathrm{BCI}=0.19,0.54)$ for 1996 and was $0.19(\mathrm{BCI}=0.091,0.33)$ for 2008.

TABLE 6. Means, medians, standard deviations (SD), and 0.025 and 0.975 quantiles for posterior distributions of parameters in the model of brucellosis in the Yellowstone bison population.

\begin{tabular}{cllllll}
\hline \hline & & & & \multicolumn{2}{c}{ Quantile } \\
\cline { 5 - 7 } Parameter & Mean & Median & SD & & 0.025 & 0.975 \\
\hline$\beta$ & 1.9 & 1.9 & 0.16 & 1.5 & 2.2 \\
$f_{\mathrm{n}}$ & 0.77 & 0.77 & 0.045 & 0.68 & 0.85 \\
$f_{\mathrm{p}}$ & 0.54 & 0.54 & 0.059 & 0.43 & 0.66 \\
$f_{\mathrm{c}}$ & 0.18 & 0.17 & 0.088 & 0.045 & 0.38 \\
$m$ & 0.47 & 0.47 & 0.041 & 0.39 & 0.55 \\
$p_{1}$ & 0.95 & 0.96 & 0.044 & 0.84 & 1.00 \\
$p_{2}$ & 0.89 & 0.88 & 0.023 & 0.84 & 0.93 \\
$p_{3}$ & 0.93 & 0.93 & 0.039 & 0.84 & 0.99 \\
$\psi$ & 0.031 & 0.027 & 0.021 & 0.004 & 0.082 \\
$\sigma_{\mathrm{p}}$ & 0.21 & 0.21 & 0.029 & 0.16 & 0.27 \\
$v$ & 0.099 & 0.081 & 0.075 & 0.0068 & 0.29 \\
& & & & &
\end{tabular}

Notes: Definitions are: $\beta$, the continuous time rate of frequency-dependent transmission $\left(\mathrm{yr}^{-1}\right) ; f_{\mathrm{n}}$, number of juveniles recruited per susceptible adult female; $f_{\mathrm{p}}$, number of juveniles recruited per recovered adult female; $f_{\mathrm{c}}$, number of juveniles recruited per infected and infectious adult female; $m$, sex ratio of juveniles surviving to yearlings; $\psi$, probability of recrudescence; $p_{1}$, juvenile survival probability; $p_{2}$, adult and yearling female survival probability; $p_{3}$, yearling and adult male survival probability; $\sigma_{\mathrm{p}}$, process standard deviation; $v$, probability of vertical transmission. 
Corresponding estimates of the probability of seropositive females with $B$. abortus detected through targeted culture of lymphatic tissue were $0.46(\mathrm{BCI}=0.28,0.64)$ for 1996 (Roffe et al. 1999) and $0.24(\mathrm{BCI}=0.16,0.31)$ for 2008 (Treanor et al. 2011). Credible intervals on outof-sample means overlapped the model estimate of means in both cases.

\section{Parameter estimates}

All parameters were estimated with reasonably narrow credible intervals (Table 6). Shrinkage of the posterior distribution relative to the prior and/or changes in its mean demonstrated that the data informed parameters beyond the information contained in the priors for all parameters except the probability of vertical transmission $v$ (Fig. 6).

Brucellosis reduced bison recruitment. The mean difference in number of offspring produced per year between a seronegative (susceptible) adult female and a seroconverting (infectious) female $\left(f_{\mathrm{n}}-f_{\mathrm{c}}\right)$ was 0.59 $(\mathrm{BCI}=0.36,0.76)$. The mean difference between a seronegative adult female and a seropositive (recovered) adult female $\left(f_{\mathrm{n}}-f_{\mathrm{p}}\right)$, was $0.22(\mathrm{BCI}=0.069,0.37)$.

\section{State variables and derived quantities}

Census data and model estimates of the posterior distributions of total population size during 1975-2010 showed a steadily increasing number of bison in Yellowstone, interspersed with declines following management removals (Fig. 7). The median estimate of population size in 1975 was $1040(\mathrm{BCI}=827,1260)$, which increased to a peak of $4865(\mathrm{BCI}=4464,5265)$ in 2005 and declined to 3805 (BCI $=3394,4214)$ in 2010.

Increases in abundance were episodically interrupted by planned management removals. Removals were strongly biased toward males during the early 1990s; 1.8 yearling and adult males were removed for every yearling and adult female. This bias switched to favor females during the 2000s; 1.3 yearling and adult females were removed for every yearling and adult male.

Brucellosis slowed the growth rate of the bison population relative to the growth rate that would be expected for a population that was brucellosis free (Fig. $8)$. The mean of the posterior distribution of the difference in growth rate between a healthy and an infected population, i.e., $\lambda$ (healthy) $-\lambda$ (infected) was 0.046 ( $\mathrm{BCI}=-0.014,0.11)$.

Brucellosis also affected the age and sex structure of the bison population, but the effects were not large. The posterior distributions of the stable stage distributions showed a reduced proportion of juveniles in an infected population relative to a brucellosis-free one and corresponding increases in the proportion of adult females, yearling females, and non-juvenile males (Table 7).

Model estimates closely tracked aerial and groundbased estimates of the sex and age composition of the population for juveniles and yearling females (Fig. 9A, B), but overestimated non-juvenile males and underestimated adult females (Fig. 9C, D). The proportion of juveniles and yearling females in the population did not depart from what would be expected from a stable age distribution of a brucellosis-free bison population experiencing exponential growth (Fig. 9A, D). However, there were more adult females and fewer yearling and adult males than would be expected for a healthy, exponentially growing population (Fig. 9C, D). The departure of the composition of the population from the distribution for a healthy population appears to have been caused in part by the disease, which depressed recruitment, causing a lower proportion of juveniles in the population and a correspondingly larger proportion of adults (Fig. 9). Male bias in removals during the 1990 s probably also contributed to the disproportionally high female segment in the population and the low male segment.

We found no clear, temporal trend in model estimates of seroprevalence of brucellosis in Yellowstone bison during 1975-2010 (Fig. 10). The median of the posterior distribution of seroprevalence in adult females declined during 1975-1985, but the $2.5 \%$ and $97.5 \%$ quantiles revealed that we could not rule out static seroprevalence during the decade. Posterior distributions of the proportion of females that were seropositive showed the effects of accumulating exposure to brucellosis as animals matured. Averaged over 1975-2010, the mean seroprevalence in juvenile females was 0.13 (BCI $=0.11$, 0.16 ) increasing to 0.23 ( $\mathrm{BCI}=0.2,0.28)$ in yearlings and $0.54(\mathrm{BCI}=0.48,0.6)$ in adult females.

Horizontal transmission was the dominant route of transmission, contributing $89 \%$ of new infections annually (Table 8). Recrudescence contributed 9.6\% (BCI = $1.7,24)$ of all conversion of disease state. Less than $1 \%$ of all transmissions occurred vertically.

We estimated that a single infectious individual in a wholly susceptible population would create a median of 1.75 new infections over its lifetime (Fig. 11). We analyzed the importance of the horizontal transmission rate, $\beta$, relative to other parameters controlling transmission, the probability of vertical transmission $(v)$ and recrudescence $(\psi)$, by setting $v$ and $\psi=0$ and recalculating $R_{0}$. Eliminating vertical transmission had a negligible effect on $R_{0}$, changing the median of the posterior distribution from 1.75 to 1.74 . In contrast, removing recrudescence reduced the median of $R_{0}$ to 1.44, indicating that chronic brucellosis plays an important role in maintaining disease in the population.

There were no discernible temporal trends in model estimates of the effective reproductive ratio $R_{\mathrm{e}(t)}$ or the annual probability of horizontal infection (Fig. 12) after 1990, although the probability of infection appeared to increase during earlier decades. Most of the mass of the posterior distribution of $R_{\mathrm{e}(t)}$ exceeded 1. This occurred because newly infected juvenile, yearling, and adult females were exposed to mortality before they became infectious. Therefore, an $R_{\mathrm{e}}$ exceeding 1 is consistent with quasi steady state in disease prevalence. 

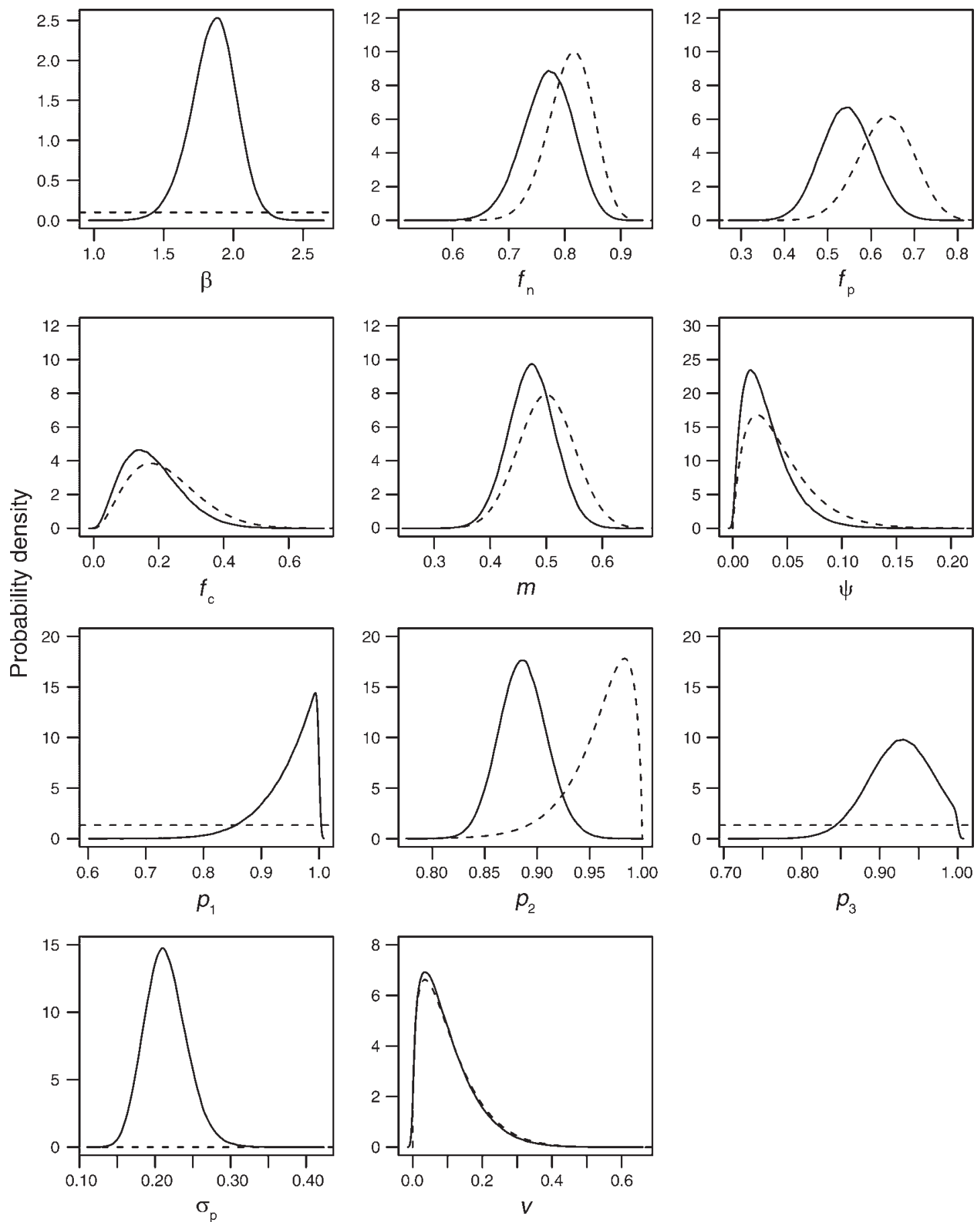

FIG. 6. Prior (dashed line) and posterior (solid line) distributions of parameters in the model of brucellosis in the Yellowstone bison population. Definitions of parameters are $\beta$, the continuous time rate of frequency-dependent transmission $\left(\mathrm{yr}^{-1}\right) ; f_{\mathrm{n}}, \mathrm{number}$ of juveniles recruited per susceptible adult female; $f_{\mathrm{p}}$, number of juveniles recruited per recovered adult female; $f_{\mathrm{c}}$, number of juveniles recruited per infected and infectious adult female; $m$, sex ratio of juveniles surviving to yearlings; $\psi$, probability of recrudescence; $p_{1}$, juvenile survival probability; $p_{2}$, adult and yearling female survival probability; $p_{3}$, yearling and adult male survival probability; $\sigma_{\mathrm{p}}$, process standard deviation; $v$, probability of vertical transmission. Note that the dashed line for the prior on $\sigma_{\mathrm{p}}$ might be difficult to see because it is slightly above zero. 


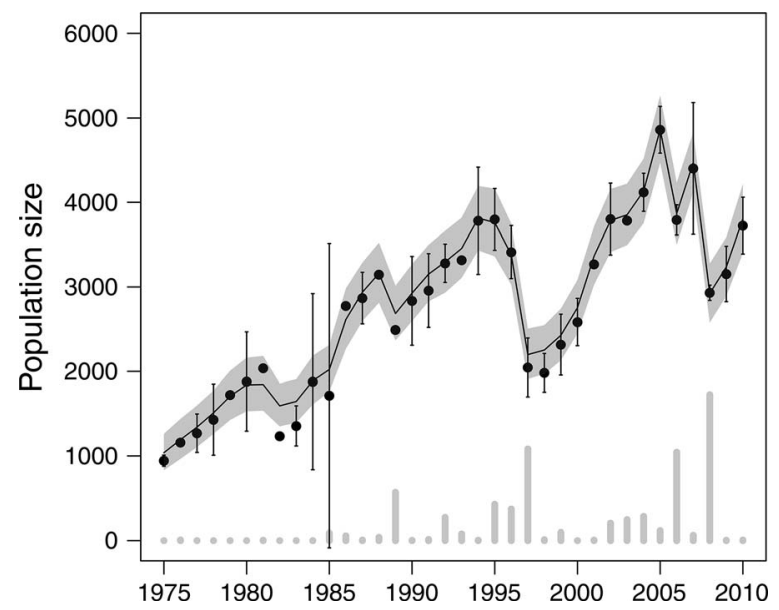

FIG. 7. Medians (solid line), and 0.025 and 0.975 quantiles (shaded area) of the posterior distributions of the number of animals in the Yellowstone bison population during 1975-2010. Model estimates are corrected for sightability. Points are means of census estimates taken during June and July \pm 2 SD. Vertical lines along the $x$-axis show the number of animals removed during the winter preceding the summer census.

The probability that a susceptible female would become infected averaged $0.12(\mathrm{BCI}=0.1,0.15)$ over all years. The proportion of adult females that were infectious (Fig. 13A) remained roughly constant, averaging 0.096 (BCI $=0.077,0.12)$ across years. The multiyear mean proportion of seropositive females that were currently in the infective stage was 0.18 (BCI = $0.14,0.22)$; see Fig. 13B.

\section{Forecasts}

We first made forecasts assuming no uncertainty in the ability to implement management. This set of forecasts is useful for comparing the ability of actions to meet goals for the population if management could be implemented exactly as specified. Uncertainty in forecasts of all quantities of interest increased as the forecast horizon extended from one to five years (Table 9). The model predicted a steady increase in population size; the 0.025 quantile for total population size in year five exceeded the median estimate in year zero for all management scenarios. The vaccination scenario allowed the largest increases in population abundance. Medians of the posterior predictive distributions for the proportion of juveniles, adult females, and non-juvenile males in the population remained relatively constant over the forecast horizon for all management alternatives, but uncertainty in the estimates increased. Median seroprevalence and probability of horizontal transmission increased for the no action, remove seronegative, and hunting scenarios, remained essentially static for the vaccination scenario, and declined markedly for the remove seropositive scenarios.

We illustrate in Fig. 14 the specific implementation of our general approach to evaluating alternatives for management (Fig. 3), using vaccination as a case example. Full summary statistics comparing management alternatives are shown in Table 10. In most cases, active management (hunting, removals, and vaccination) failed to increase the probability of meeting management goals five years into the future relative to no action. There were exceptions, however. Removals of seronegative and seropositive females and hunting increased the probability of maintaining the population below 3500 by at least 29 -fold relative to no action. By year five, removing seropositive females improved the probability of reducing seroprevalence below $40 \%$ by 30 fold relative to no action and was 120 times more likely to reduce transmission probability by half relative to no action. There was a 0.64 probability that removing seropositives would cut transmission probability by half in five years and a 0.87 probability that seroprevalence would be reduced below $40 \%$. Vaccination increased the probability of reducing seroprevalence below $40 \%$ by fivefold and increased the probability of achieving a $50 \%$ reduction in transmission probability by a factor of 2 relative to no action. The probability of meeting goals for seroprevalence and transmission was less than 0.07 using vaccination, but there was evidence that vaccination would be more likely to move the population toward the goal than the no-action alternative.

We also made forecasts reflecting uncertainty arising from year-to-year variation in the ability to hunt, remove, or treat animals crossing the park boundary (Table 11). When management uncertainty was included in forecasts, the probability of meeting management goals was substantially reduced for almost all action (Table 12) relative to estimates that did not include management uncertainty. There were only a few management alternatives that were more likely to meet goals than no action. Hunting increased the probability of maintaining the population below 3500 by 170 -fold and showed a reasonable probability $(0.24)$ of meeting that goal. Vaccination caused a 1.7 times increase in the probability of meeting goals for reducing the probability of horizontal transmission to half its pretreatment value in five years, but the absolute probability of meeting that goal was essentially 0 (Table 12). Removing seropositives was the only management action that showed promise for achieving goals for disease management, increasing the probability of meeting goals for seroprevalence by 4.8 -fold relative to no action and by 2.6 times for reducing transmission probability by half in year 5 . However, the probability of reaching these goals was low, 0.14 for seroprevalence and 0.014 for probability of transmission.

Virtually all actions reduced the probability of meeting goals for population sex and age composition relative to the no-action alternative. This shows the potential for trade-offs that can occur when an action moves the population toward one desired state but away from another. For example, removing seropositive females increased the probability of meeting goals for 


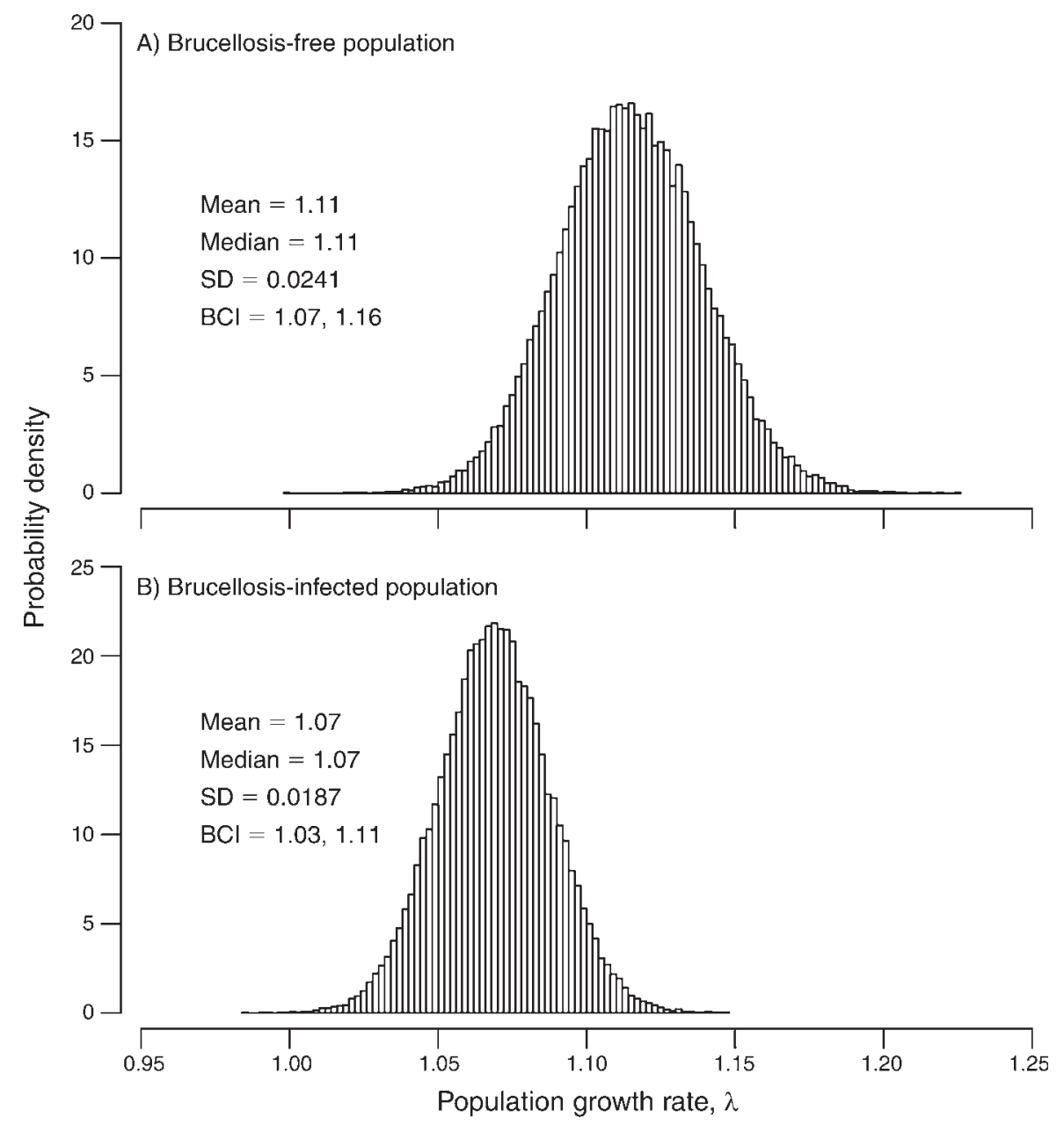

FIG. 8. Posterior distributions of population growth rate of (A) a brucellosis-free and (B) a brucellosis-infected population of bison in Yellowstone. The brucellosis-free population was modeled with recruitment rates for susceptible animals and a zero probability of exposure. The brucellosis-infected population was modeled using estimated parameters for all recruitment rates. Infection probability was estimated using a draw from the model estimate of the distribution of average infection probability during 1975-2010. BCI is the equal-tailed $95 \%$ Bayesian credible interval ( 0.025 and 0.975 quantiles).

seroprevalence and probability of infection, but by reducing the number of females, reduced the probability of meeting goals for the proportion of females in the population relative to no action.

\section{Discussion}

Bayesian state-space modeling in support of adaptive management

Carl Walters (1986) encouraged the use of Bayesian models to support decisions on adaptive management in his seminal book, Adaptive management of renewable resources. The Bayesian approach to inference and the adaptive approach to management are natural allies: both embody updating current knowledge with new data. The modeling framework that we used to support management of the Yellowstone bison is relevant to many contemporary problems in environmental policy and management. These problems share four elements: a
TABLE 7. Estimates of stable stage distribution for a brucellosis-free bison population and a population infected with brucellosis, where the infection probability is drawn from the distribution of the average infection probability during 19752010.

\begin{tabular}{llll}
\hline \hline & & \multicolumn{2}{c}{ Quantile } \\
\cline { 4 - 4 } Status and stage & Median & 0.025 & 0.975 \\
\hline Infected & & & \\
Calves & 0.15 & 0.099 & 0.18 \\
Yearling females & 0.068 & 0.046 & 0.087 \\
Adult females & 0.33 & 0.22 & 0.4 \\
Non-calf males & 0.46 & 0.34 & 0.63 \\
Healthy & & & \\
Calves & 0.19 & 0.15 & 0.22 \\
Yearling females & 0.083 & 0.064 & 0.1 \\
Adult females & 0.31 & 0.25 & 0.36 \\
Non-calf males & 0.42 & 0.33 & 0.54 \\
\hline
\end{tabular}



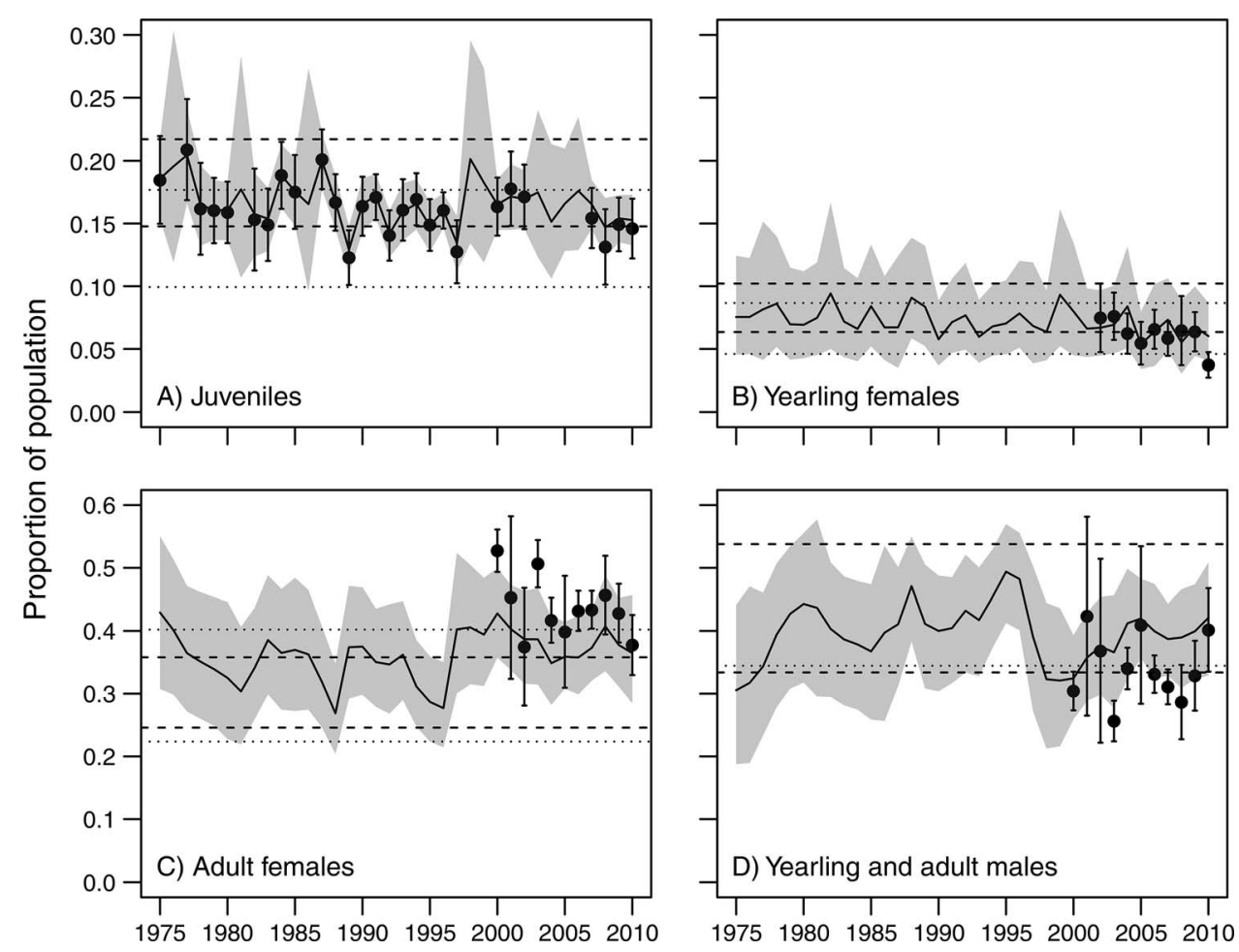

FIG. 9. Medians (solid line), and 0.025 and 0.975 quantiles (shaded area) of the posterior distributions of the proportions of the Yellowstone bison population in modeled age and sex classes. Points are means \pm 2 SD of annual classification counts. Horizontal dashed lines delimit BCIs for the proportion of each age and sex class in a stable age distribution of a healthy, exponentially growing bison population. Horizontal dotted lines delimit BCIs for the proportion of each age and sex class in an infected population assuming a transmission probability drawn from the distribution of the average infection probability during 1975-2010 (median $=0.19, \mathrm{BCI}=16,0.23$ ).

history of monitoring providing time series data, targeted research studies of key processes, and a need to combine all sources of information in a modeling framework that is honest about uncertainty.

As originally conceived, adaptive management depends on the application of Bayesian models to examine alternatives for policy and management (Walters 1986). We describe an approach to evaluating alternative courses of action in terms of their ability to meet specific goals for the system being managed (Fig. 3). The essence of this problem is to compare the value of taking some specific action with a null model of no action. Actions that merit implementation should increase the probability of meeting goals relative to no action, given the current state of knowledge and uncertainty. Note that this approach allows us to conclude that a particular action is superior to the no-action alternative, even when the absolute probability of meeting a goal given the action remains relatively low. As uncertainty increases, it becomes less likely that management actions will have outcomes superior to the no-action approach, a result that properly reinforces caution in management. Comparing take-action alternatives to the null model of no action can improve the conversation among policy makers, managers, and stakeholders seeking to solve a broad range of environmental problems.

\section{Managing Yellowstone bison}

Properly partitioning process and observation variance enabled our model to offer true forecasts: predictions of the future state accompanied by properly specified uncertainty. Errors in our forecasts propagated with time as they should, reducing confidence about the true state as the forecast horizon extended into the future. Other models have been used to support decisions on managing brucellosis in the Yellowstone bison (Peterson et al. 1991, Dobson and Meagher 1996, Angliss 2003). We build on this earlier work by differentiating the uncertainties in the data, in estimates of parameters, and in the mathematical representation of the disease process.

We show that these uncertainties combine to assure that long-term predictions, e.g., 20 years in Peterson et al. (1991); 35 years in Ebinger et al. (2011); 30 years in Treanor et al. (2010) will be unreliable because credible intervals on forecasts expand rapidly with increases in the forecast horizon (Table 9). Long-range forecasts will include an enormous range of probable outcomes. This finding urges caution in making long-term forecasts with ecological models. The uncertainties inherent in modeling brucellosis in the Yellowstone bison emphasize the value of adaptive management implemented over 


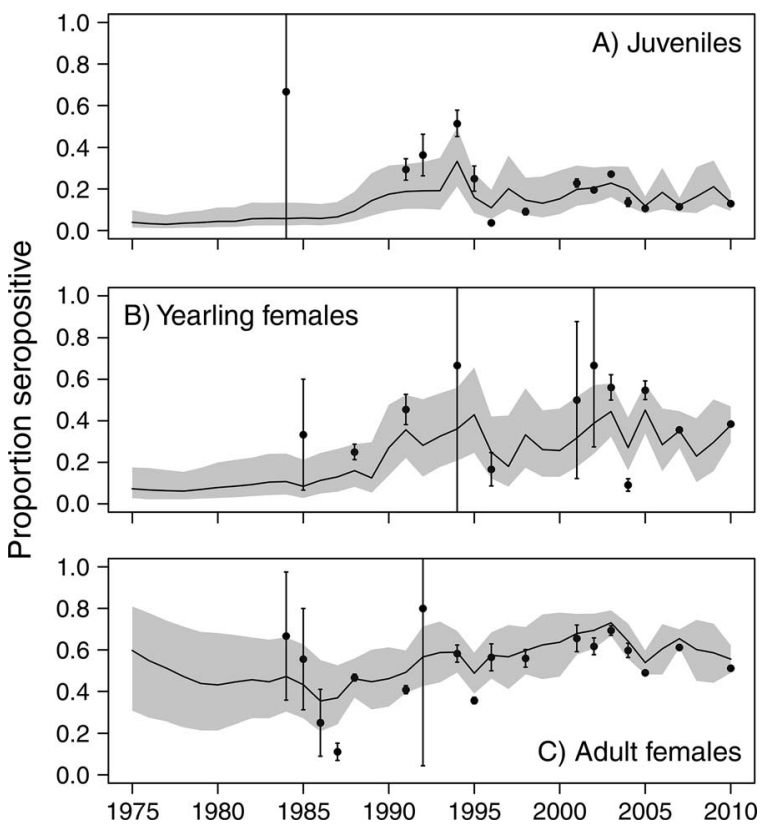

FIG. 10. Medians (solid line), and 0.025 and 0.975 quantiles (shaded area) of the posterior distributions of model estimates of the proportion of females in the Yellowstone bison population that were seropositive for brucellosis. Points are means from observations of serological status $\pm 2 \mathrm{SD}$ (vertical bars).

relatively brief decision horizons, where models and decisions are updated frequently in an iterative cycle.

In virtually all problems of environmental management, there are multiple goals for a desired state of the system. Management of Yellowstone bison seeks to reach specific outcomes for population size, demography, and disease states and processes. Our intent was not to explore an exhaustive list of management alternatives, but to generally compare the efficacy of hunting, selective removal, and vaccination for meeting management goals. Population size can be controlled using a combination of out-of-park hunting and boundary removals, as has been shown during the past three decades. Thus, the question posed to managers is whether selective removal and/or vaccination can be used to change the management strategy for the conservation of bison. Eradication of brucellosis is probably needed to expand the conservation area of bison well beyond areas surrounding Yellowstone (Cheville et al. 1998). More realistically, eradication would preclude any potential spillover from bison to livestock, but probably would not dramatically change the ultimate area that bison are tolerated outside the park due to other social concerns about living with wild bison (Plumb et al. 2009). When we increased uncertainty in forecasts by adding stochastic variation in the number of animals that could be annually hunted, removed, or vaccinated, we found low probability of meeting desired outcomes for brucellosis suppression under all considered management alternatives.
This result is not as pessimistic as it might appear. One of the primary uses of a model like this one is to improve the conversation between stakeholders and managers. The model can be valuable in helping managers and citizens arrive at realistic goals and to realize that there will be inherent risks associated with meeting those goals. For example, our analysis shows that reducing the probability of transmission by one half in five years using vaccination is not likely when we include uncertainty in the ability of managers to treat a targeted number of seronegative females. Forecasts suggested that there was virtually no chance of meeting that goal (Table 12). Similarly there was a $7 \%$ chance of reducing adult female seroprevalence below $40 \%$ using vaccination. We can nonetheless use this work to articulate what level of brucellosis suppression is feasible given current technology. For example, managers and stakeholders might agree that it is enough to be moving in the right direction with efforts to reduce risk of infection from brucellosis. In this case, a reasonable goal might be "Reduce the probability of exposure by $10 \%$ relative to the current median value." The odds of meeting that goal using vaccination increased to $26 \%$. With this less ambitious goal, vaccination increases the probability that the goal would be met relative to no action by a factor of only 1.4. This illustrates a fundamental trade-off in making management choices in the face of uncertainty: less ambitious goals are more likely to be met, but they offer smaller improvements in the probability of obtaining the desired outcome relative to no action.

It follows that a key to management of brucellosis in bison is to choose goals and actions that reduce the risk of undesirable outcomes, particularly in transmission to livestock on lands surrounding the park, rather than attempting to achieve goals that are simply not attainable. Separation of bison and livestock outside of Yellowstone through fencing and intensive hazing of bison has been $100 \%$ successful at preventing spillover of brucellosis from bison to cattle (White et al. 2011). Further, selective removal of seropositive females and/or vaccination may marginally reduce $B$. abortus preva-

TABLE 8. Proportional contributions of vertical and horizontal transmission to new infections.

\begin{tabular}{llll}
\hline \hline \multirow{2}{*}{\multicolumn{1}{c}{ Transmission }} & & \multicolumn{2}{c}{ Quantile } \\
\cline { 3 - 4 } \cline { 3 - 4 } & Median & 0.025 & 0.975 \\
\hline Vertical from seroconverter & 0.0055 & 0.00037 & 0.029 \\
Vertical from recrudesced & 0.002 & 0.00011 & 0.011 \\
Horizontal juvenile & 0.31 & 0.25 & 0.37 \\
Horizontal yearling & 0.17 & 0.13 & 0.19 \\
Horizontal adult & 0.41 & 0.33 & 0.47 \\
All vertical & 0.0081 & 0.00062 & 0.036 \\
All horizontal & 0.89 & 0.74 & 0.98 \\
Recrudesence & 0.096 & 0.017 & 0.24 \\
\hline
\end{tabular}

Note: Recrudescence gives the number of infectious recovered animals divided by the total number of conversions of disease state. 


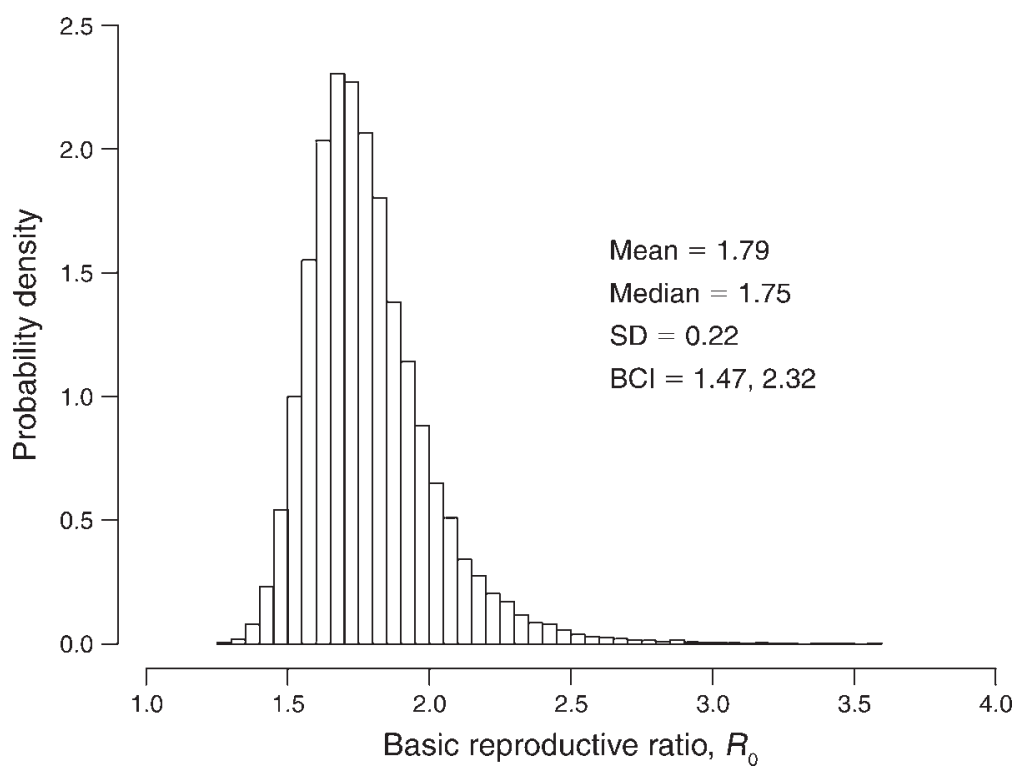

FIG. 11. Posterior distributions of the basic reproductive ratio $\left(R_{0}\right)$ for brucellosis in the Yellowstone bison population. The estimates are the number of new infections created by a single infected individual in an otherwise healthy population of 3000 individuals with a sex-age distribution of an exponentially growing, brucellosis-free population. BCI is the equal-tailed $95 \%$ Bayesian credible interval ( 0.025 and 0.975 quantiles).
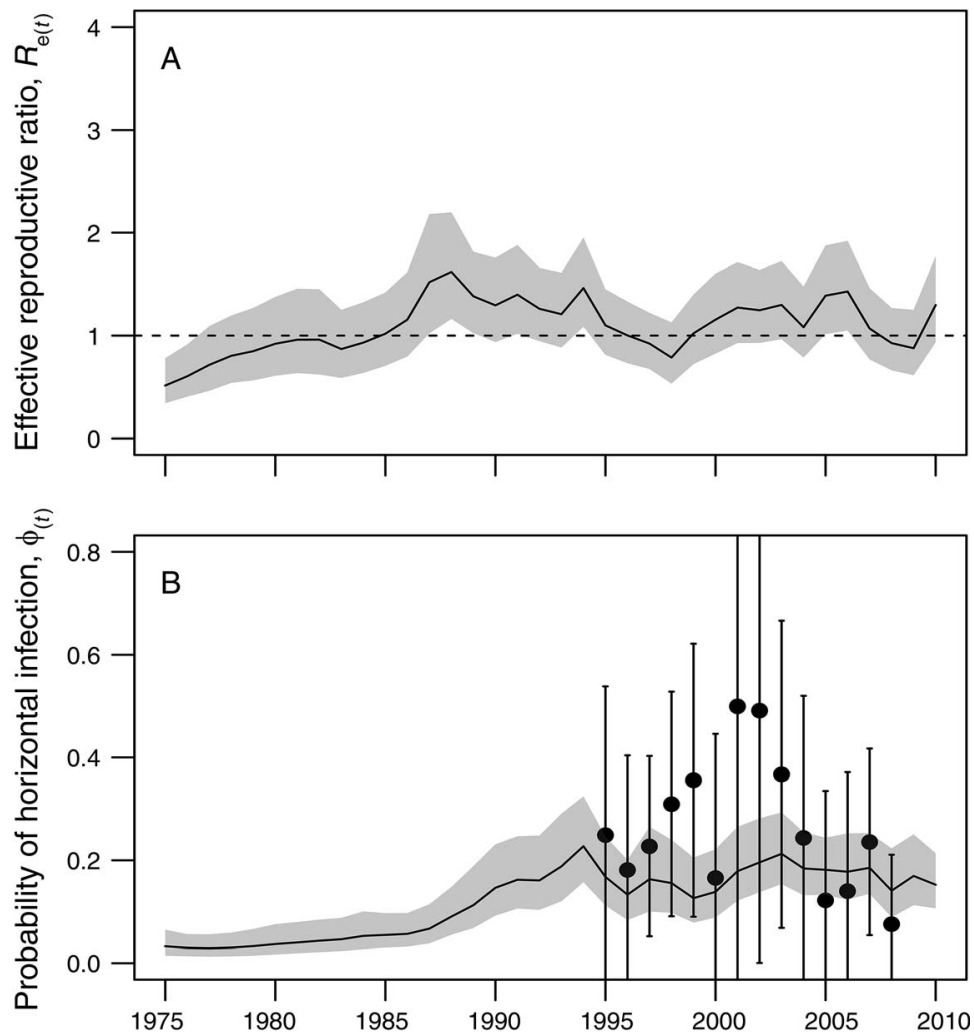

FIG. 12. Medians (solid line), and 0.025 and 0.975 quantiles (shaded area) of the posterior distributions of model estimates of (A) the effective reproductive ratio $\left(R_{\mathrm{e}(t)}\right)$ and $(\mathrm{B})$ the annual probability $\left(\phi_{(t)}\right)$ that a susceptible bison will become infected via horizontal transmission for the years 1975-2010. Points in panel (B) are estimates ( \pm 2 SD) of infection probability derived from mark-recapture studies that were not used to estimate model parameters. 
lence and the probability of exposure due to variations in the timing and extent of out-of-park migrations. Undoubtedly, large numbers of bison will be removed from the Yellowstone population over time in order to maintain abundance within desired conditions. Thus, it is important to make informed decisions to protect demographic and genetic characteristics of the population. Results of our model can be used to support collaborative approaches to conservation where stakeholders and managers make informed choices of realistic goals and actions to meet them, appreciating that success will be uncertain.

\section{Understanding brucellosis in the Yellowstone bison}

Brucellosis is a chronic condition of the Yellowstone bison population that appears to have reached a quasisteady state. Estimates of the true serological state of the population showed no temporal trend in any age class during the last two decades (Fig. 10). The median, true percentage of the population of adult females that were infectious remained close to $9.6 \%$ for the last 35 years with no directional change over that interval, a finding that resembles the earlier results for the percentage that is infectious $(\sim 10 \%)$ reported by Dobson and Meagher (1996). For the last three decades, the effective reproductive ratio was slightly above 1.0, suggesting that the disease has reached an approximate equilibrium in the population (Fig. 12A), given the lag time between the time animals are exposed and become infectious. Credible intervals on the annual probability that a susceptible animal becomes infected via horizontal transmission failed to reveal any increasing or decreasing temporal trend during 1975-2010 (Fig. 12B). This endemic equilibrium results from the reduction in transmission from each infectious individual as the proportion of bison that acquire immunological resistance increased over time.

This relative stasis in the state of brucellosis infection, and the processes governing it, occurred despite large perturbations to the population. A total of 6810 bison were removed from the population by gather and slaughter during 1985-2010. Some of these annual removals reduced the population dramatically (e.g., by $31 \%$ in $1997,21 \%$ in 2006 , and $39 \%$ in 2008). If transmission depended on density, we would expect removals of this magnitude to substantially reduce the probability of horizontal transmission, which we did not observe in the data (Fig. 12). Sixty-six percent of females removed were seropositive, a fraction that did not differ from the serological composition of the population of females (Fig. 10B). Thus, it is likely that removals failed to cause substantial reductions in the proportion of the population that was infectious, and consequently, we would not expect marked changes in probability of frequency dependent transmission or changes in the proportion of the population that was seropositive in response to removals. It follows that the pattern of removals and the relative stasis in brucellosis stages and

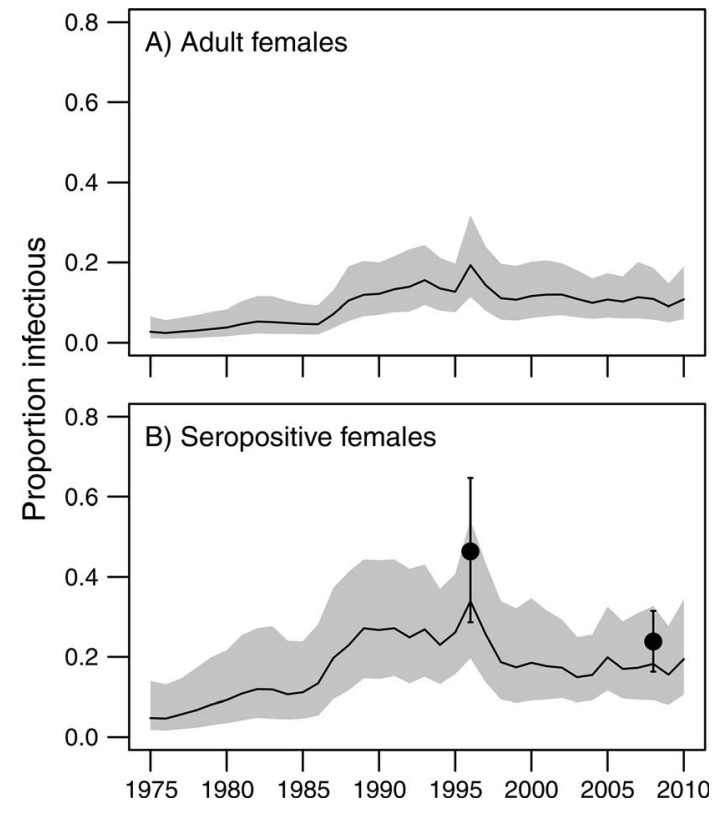

FIG. 13. Medians (solid line), and 0.025 and 0.975 quantiles (shaded area) of the posterior distributions of model estimates of (A) the proportion of the population of adult female bison in Yellowstone that were infectious for brucellosis and (B) the proportion of the seropositive females that were infectious; the two points are independent estimates (mean with $95 \%$ equaltailed BCI) of the proportion of seropositive animals with detectable Brucella abortus through culture of targeted lymphatic tissue (Roffe et al. 1999, Treanor et al. 2011). These estimates were not used to fit the model.

processes is more consistent with a frequency-dependent mechanism of transmission than a density-dependent one.

This inference is reinforced by model selection that provided reasonable evidence for frequency-dependent transmission of brucellosis in the Yellowstone bison population relative to the other transmission models (section Model evaluation and selection), a finding consistent with observations of bison social behavior and patterns of movement. Bison aggregate in large herds during the calving season when transmission occurs, and the density of these groups does not appear to increase with population size, a requirement for density-dependent transmission. The hallmark of frequency dependence is that population density does not increase as population size increases, allowing contact rates to depend on the proportion of the population that is infectious rather than the number of infectious individuals in the population. We would expect frequency dependence to prevail if the area used by the population expands as the population size increases, allowing density to remain constant. There is ample evidence that bison expanded the area of habitat used after 1975 (Gates et al. 2005, Bruggeman et al. 2006, Plumb et al. 2009). Dobson and Meagher (1996) also found that a continuous-time compartment model with frequency-dependent transmission provided better qual- 
TABLE 9. Five-year forecasts (year 0 is the last year with data) of the median of the posterior predictive distribution (95\% Bayesian credible interval in parentheses) for population states and horizontal transmission probability under different management scenarios, assuming no uncertainty in the ability to implement management.

\begin{tabular}{|c|c|c|c|c|}
\hline Scenario and goal & Year 0 & Year 1 & Year 3 & Year 5 \\
\hline \multicolumn{5}{|l|}{ No action } \\
\hline Juveniles & $0.15(0.13,0.17)$ & $0.18(0.11,0.26)$ & $0.16(0.094,0.25)$ & $0.16(0.089,0.25)$ \\
\hline Males & $0.41(0.32,0.5)$ & $0.4(0.27,0.55)$ & $0.42(0.25,0.61)$ & $0.42(0.24,0.64)$ \\
\hline Adult females & $0.37(0.29,0.46)$ & $0.34(0.24,0.46)$ & $0.34(0.22,0.47)$ & $0.34(0.2,0.48)$ \\
\hline Total population & $3830(3420,4220)$ & $4310(3400,5530)$ & $5300(3620,8010)$ & $6510(3980,11100)$ \\
\hline Seroprevalence & $0.55(0.49,0.62)$ & $0.59(0.44,0.73)$ & $0.61(0.4,0.79)$ & $0.63(0.39,0.81)$ \\
\hline $\operatorname{Pr}(\operatorname{transmission})$ & $0.13(0.084,0.19)$ & $0.13(0.082,0.21)$ & $0.14(0.079,0.23)$ & $0.15(0.081,0.26)$ \\
\hline \multicolumn{5}{|l|}{ Remove seropositives } \\
\hline Juveniles & $0.15(0.13,0.17)$ & $0.17(0.11,0.26)$ & $0.14(0.075,0.24)$ & $0.14(0.065,0.25)$ \\
\hline Males & $0.41(0.32,0.5)$ & $0.43(0.29,0.58)$ & $0.49(0.29,0.7)$ & $0.52(0.29,0.76)$ \\
\hline Adult females & $0.37(0.29,0.46)$ & $0.32(0.22,0.43)$ & $0.29(0.16,0.43)$ & $0.26(0.12,0.42)$ \\
\hline Total population & $3830(3420,4220)$ & $4030(3150,5220)$ & $4290(2750,6800)$ & $4640(2520,8710)$ \\
\hline Seroprevalence & $0.55(0.49,0.62)$ & $0.52(0.37,0.67)$ & $0.35(0.097,0.62)$ & $0.14(0.0012,0.57)$ \\
\hline $\operatorname{Pr}(\operatorname{transmission})$ & $0.13(0.084,0.19)$ & $0.11(0.069,0.18)$ & $0.087(0.027,0.17)$ & $0.041(0,0.16)$ \\
\hline \multicolumn{5}{|l|}{ Remove seronegatives } \\
\hline Juveniles & $0.15(0.13,0.17)$ & $0.16(0.1,0.25)$ & $0.13(0.067,0.22)$ & $0.12(0.052,0.21)$ \\
\hline Males & $0.41(0.32,0.5)$ & $0.44(0.29,0.59)$ & $0.5(0.3,0.71)$ & $0.55(0.31,0.78)$ \\
\hline Adult females & $0.37(0.29,0.46)$ & $0.32(0.22,0.44)$ & $0.29(0.16,0.44)$ & $0.26(0.12,0.43)$ \\
\hline Total population & $3830(3420,4220)$ & $3990(3110,5160)$ & $4110(2600,6570)$ & $4160(2200,8000)$ \\
\hline Seroprevalence & $0.55(0.49,0.62)$ & $0.67(0.52,0.79)$ & $0.8(0.57,0.96)$ & $0.92(0.63,1)$ \\
\hline $\operatorname{Pr}(\operatorname{transmission})$ & $0.13(0.084,0.19)$ & $0.15(0.091,0.23)$ & $0.16(0.09,0.27)$ & $0.16(0.08,0.28)$ \\
\hline \multicolumn{5}{|l|}{ Hunting } \\
\hline Juveniles & $0.15(0.13,0.17)$ & $0.18(0.11,0.26)$ & $0.16(0.093,0.25)$ & $0.16(0.088,0.25)$ \\
\hline Males & $0.41(0.32,0.5)$ & $0.4(0.27,0.55)$ & $0.42(0.25,0.61)$ & $0.42(0.24,0.64)$ \\
\hline Adult females & $0.37(0.29,0.46)$ & $0.34(0.24,0.46)$ & $0.34(0.22,0.47)$ & $0.34(0.2,0.47)$ \\
\hline Total population & $3830(3420,4220)$ & $4070(3200,5220)$ & $4500(2970,6960)$ & $5020(2850,9010)$ \\
\hline Seroprevalence & $0.55(0.49,0.62)$ & $0.59(0.44,0.73)$ & $0.61(0.4,0.79)$ & $0.63(0.39,0.81)$ \\
\hline $\operatorname{Pr}($ transmission $)$ & $0.13(0.084,0.19)$ & $0.13(0.082,0.21)$ & $0.14(0.08,0.23)$ & $0.15(0.081,0.26)$ \\
\hline \multicolumn{5}{|l|}{ Vaccination } \\
\hline Juveniles & $0.15(0.13,0.17)$ & $0.18(0.11,0.26)$ & $0.16(0.096,0.25)$ & $0.16(0.091,0.26)$ \\
\hline Males & $0.41(0.32,0.5)$ & $0.4(0.27,0.55)$ & $0.41(0.25,0.6)$ & $0.42(0.24,0.63)$ \\
\hline Adult females & $0.37(0.29,0.46)$ & $0.4(0.3,0.52)$ & $0.46(0.3,0.62)$ & $0.46(0.29,0.65)$ \\
\hline Total population & $3830(3420,4220)$ & $4310(3410,5510)$ & $5320(3680,7960)$ & $6600(4120,11000)$ \\
\hline Seroprevalence & $0.55(0.49,0.62)$ & $0.58(0.44,0.7)$ & $0.57(0.39,0.74)$ & $0.56(0.36,0.74)$ \\
\hline $\operatorname{Pr}($ transmission $)$ & $0.13(0.084,0.19)$ & $0.14(0.082,0.21)$ & $0.13(0.075,0.21)$ & $0.12(0.064,0.2)$ \\
\hline
\end{tabular}

Notes: States are the proportion of juveniles, adult and yearling males, and adult females in the population, total population size, and the proportion of adult females that are seropositive. The five management alternatives are: no action; annually removing 200 seropositive yearling or adult females; annually removing 200 seronegative yearling or adult females; annually harvesting 200 animals from all sex and age classes; and annually vaccinating 200 seronegative yearling or adult females. Goals are to: (1) maintain the composition of the population within the 0.025 and 0.975 quantiles for the stable age distribution of juveniles, adult females, and non-juvenile males in a brucellosis-free, exponentially growing population; (2) maintain the population at levels between 3000 and 3500 individuals; (3) reduce the seroprevalence in the population to $<40 \%$; and (4) reduce the probability of horizontal transmission to half of its value at time 0 .

itative agreement with serology data than a model with density-dependent transmission.

We are the first to estimate the basic reproductive ratio $\left(R_{0}\right)$ of brucellosis and the probability of horizontal transmission, the discrete-time expression of the force of infection. Our estimate of $R_{0}$ includes proper estimates of uncertainty, which is rare for estimates in wildlife. We know of only one other instance where this has been done (Miller et al. 2006). Credible intervals for $R_{0}(1.47,2.32)$ are not markedly different from the point estimate $\sim 2.5$ coarsely derived from assuming that $40 \%$ of the adult females are susceptible in a steady-state population (Anderson and May 1991). Our $R_{0}$ for brucellosis resembles estimates for other diseases in wildlife; for example, raccoon rabies (Coyne et al. 1989), fox rabies, bovine tuberculosis in possums (Trichosurus vulpecula, Barlow 1991) and badgers (Meles meles, Anderson and Trewhella 1985), and chronic wasting disease in mule deer Odocoileus hemionus (Miller et al. 2006).

There have been very few estimates of parameters controlling the dynamics of brucellosis. We found important roles of the acute and chronic stages of brucellosis in maintaining the disease in Yellowstone bison. Although chronic brucellosis and the re-emergence of clinical symptoms is well documented, most infected animals recover, clear the infection, and exhibit prolonged immunity to brucellosis (Ficht 2003), as indicated by the relatively low median probability of recrudescence $(0.031, \mathrm{BCI}=0.004,0.082)$. Nonetheless, eliminating recrudescence as a source of infectious animals reduced the median estimate of $R_{0}$ from 1.75 

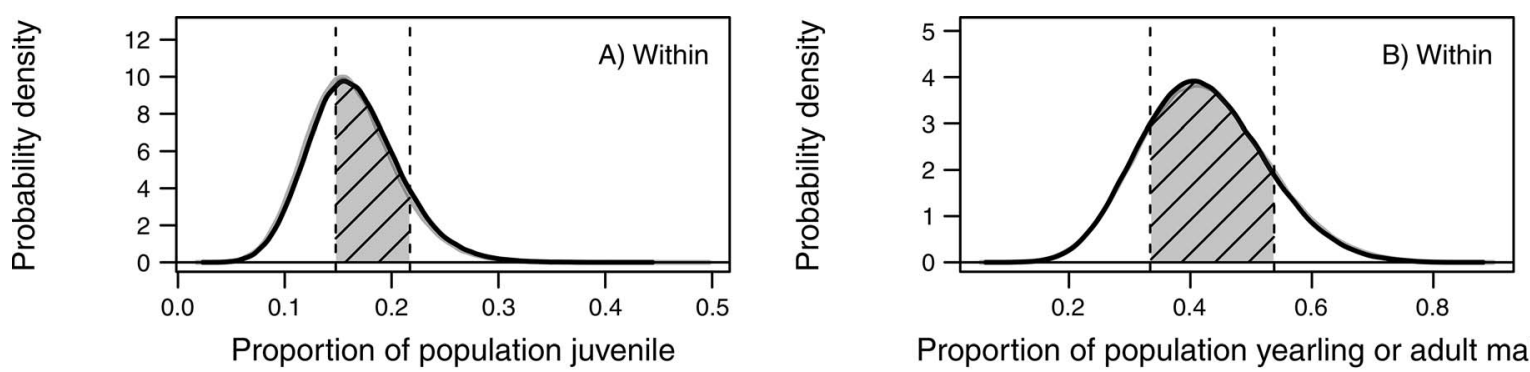

Proportion of population yearling or adult male

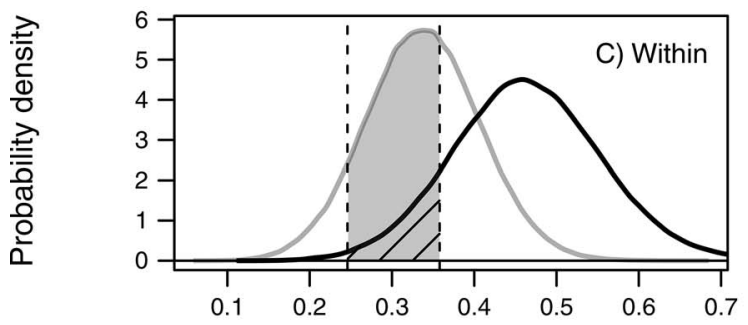

Proportion of population adult female
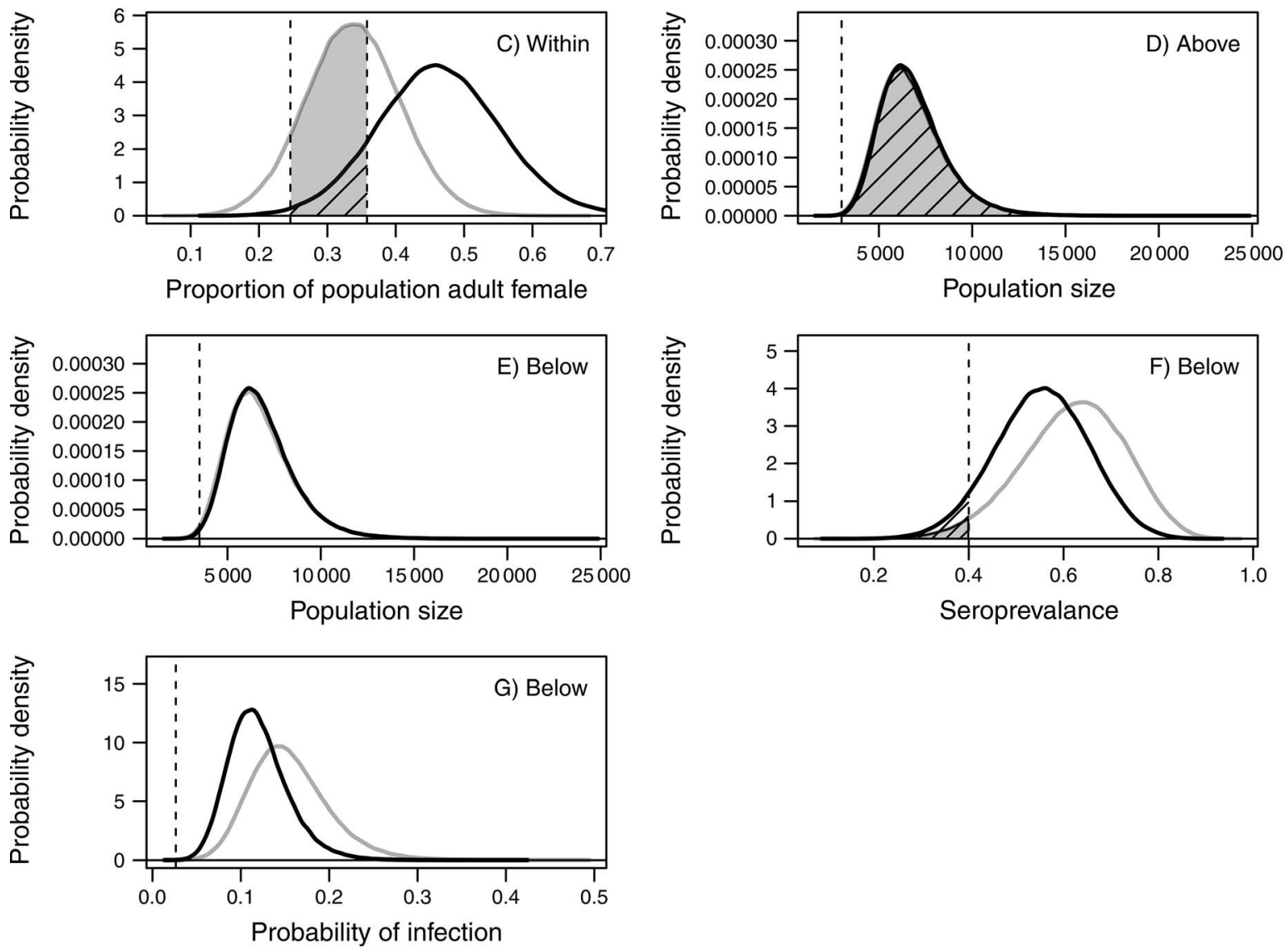

Fig. 14. Illustration of forecasts used to evaluate alternatives for management described in Fig. 3. Management goals for the state of the population are shown by vertical dashed lines. The gray curves are posterior process distributions forecasting different states five years into the future, assuming no management; the black curves show forecasts assuming that 200 seronegative animals are vaccinated annually. The probability of meeting a management goal, given no action, is shown by the shaded area. The probability of a goal, given vaccination, is shown by the hatched area. The effect of management is the ratio of the hatched area to the shaded area. The probability of meeting a goal is essentially zero if there is no shaded or hatched area visible. Panels show the ability of vaccination to meet the following goals: (1) to maintain demographic structure consistent with an unmanaged population (A-C); (2) to maintain population size above 3000 animals and below 3500 (D, E); (3) to reduce seroprevalence below $40 \%$ (F); and (4) to reduce probability of infection by half $(G)$.

to 1.44 because most animals are infectious early in life and live for a long time in the recovered state.

Our assumption of direct transition from the infectious to the recovered state may be overly simplistic. Rhyan et al. (2009) found that mature female bison observed transitioning from the exposed to the infective stages exhibited reproductive failures for up to four successive pregnancies and some were culture positive for at least two successive years.
Further, Rhyan et al. (2009) were unable to culture $B$. abortus for up to four years from adult females that entered their study seropositive, suggesting long-term immunity. Treanor et al. (2011) found that the majority of females were exposed to B. abortus by reproductive maturity and the probability of culturing B. abortus from seropositive adult female bison was dramatically reduced after five years of age. These findings suggest a prolonged infectious period that 
TABLE 10. Probability of meeting goals for the Yellowstone bison population and (in parentheses) the proportional change in probability of meeting the goal relative to taking no action, with no uncertainty in the ability to implement management.

\begin{tabular}{|c|c|c|c|c|}
\hline Scenario and goal & Year 0 & Year 1 & Year 3 & Year 5 \\
\hline \multicolumn{5}{|l|}{ No action } \\
\hline Juveniles & 0.99 & $0.5(1)$ & $0.63(1)$ & $0.61(1)$ \\
\hline Males & 0.94 & $0.79(1)$ & 0.77 (1) & $0.74(1)$ \\
\hline Females & 0.78 & $0.84(1)$ & $0.8(1)$ & $0.78(1)$ \\
\hline$N>3000$ & 0.94 & $0.96(1)$ & $0.98(1)$ & 0.99 (1) \\
\hline$N<3500$ & 0.00 & $0.0015(1)$ & $0.002(1)$ & $0.0013(1)$ \\
\hline Seroprevalence & 0.00 & $0.006(1)$ & $0.026(1)$ & 0.029 (1) \\
\hline $\operatorname{Pr}($ transmission $)$ & 0.00 & $0.0033(1)$ & $0.0052(1)$ & $0.0051(1)$ \\
\hline \multicolumn{5}{|l|}{ Remove seropositives } \\
\hline Juveniles & 0.99 & $0.58(1.2)$ & $0.67(1.1)$ & $0.6(0.98)$ \\
\hline Males & 0.94 & $0.87(1.1)$ & $0.83(1.1)$ & $0.74(1)$ \\
\hline Females & 0.78 & $0.9(1.1)$ & $0.77(0.97)$ & $0.64(0.82)$ \\
\hline$N>3000$ & 0.94 & $0.87(0.91)$ & $0.82(0.83)$ & $0.82(0.83)$ \\
\hline$N<3500$ & 0.00 & $0.0099(6.7)$ & $0.056(28)$ & $0.079(61)$ \\
\hline Seroprevalence & 0.00 & $0.066(11)$ & $0.63(24)$ & $0.87(30)$ \\
\hline $\operatorname{Pr}(\operatorname{transmission})$ & 0.00 & $0.014(4.2)$ & $0.23(45)$ & $0.64(120)$ \\
\hline \multicolumn{5}{|l|}{ Remove seronegatives } \\
\hline Juveniles & 0.99 & $0.65(1.3)$ & $0.68(1.1)$ & $0.58(0.95)$ \\
\hline Males & 0.94 & $0.88(1.1)$ & $0.81(1.1)$ & $0.68(0.92)$ \\
\hline Females & 0.78 & 0.89 (1.1) & $0.77(0.97)$ & $0.63(0.81)$ \\
\hline$N>3000$ & 0.94 & $0.85(0.89)$ & $0.76(0.77)$ & $0.71(0.71)$ \\
\hline$N<3500$ & 0.00 & $0.012(8.4)$ & $0.085(43)$ & $0.15(120)$ \\
\hline Seroprevalence & 0.00 & $0.00041(0.068)$ & $0.00074(0.029)$ & $0.00054(0.019)$ \\
\hline $\operatorname{Pr}(\operatorname{transmission})$ & 0.00 & $0.0013(0.38)$ & $0.002(0.39)$ & $0.0068(1.3)$ \\
\hline \multicolumn{5}{|l|}{ Hunting } \\
\hline Juveniles & 0.99 & $0.51(1)$ & $0.64(1)$ & $0.61(1)$ \\
\hline Males & 0.94 & $0.8(1)$ & $0.77(1)$ & $0.74(1)$ \\
\hline Females & 0.78 & $0.85(1)$ & $0.8(1)$ & $0.78(1)$ \\
\hline$N>3000$ & 0.94 & $0.89(0.93)$ & $0.89(0.9)$ & $0.9(0.9)$ \\
\hline$N<3500$ & 0.00 & $0.0068(4.6)$ & $0.027(14)$ & $0.037(29)$ \\
\hline Seroprevalence & 0.00 & $0.0063(1.1)$ & $0.026(1)$ & 0.029 (1) \\
\hline $\operatorname{Pr}($ transmission $)$ & 0.00 & $0.0033(1)$ & $0.005(0.97)$ & $0.0053(1)$ \\
\hline \multicolumn{5}{|l|}{ Vaccination } \\
\hline Juveniles & 0.99 & $0.5(1)$ & $0.63(0.99)$ & $0.59(0.96)$ \\
\hline Males & 0.94 & $0.8(1)$ & $0.77(1)$ & $0.74(1)$ \\
\hline Females & 0.78 & $0.5(0.59)$ & $0.24(0.31)$ & $0.24(0.31)$ \\
\hline$N>3000$ & 0.94 & $0.96(1)$ & 0.99 (1) & $1(1)$ \\
\hline$N<3500$ & 0.00 & $0.0014(0.96)$ & $0.0015(0.73)$ & $0.00068(0.53)$ \\
\hline Seroprevalence & 0.00 & $0.0053(0.89)$ & $0.03(1.2)$ & $0.063(2.2)$ \\
\hline $\operatorname{Pr}($ transmission $)$ & 0.00 & $0.0032(0.98)$ & $0.0076(1.5)$ & $0.026(5.2)$ \\
\hline
\end{tabular}

Notes: The forecast horizon is five years, where year 0 is the final year with data. The five management alternatives are: no action; annually removing 200 seropositive yearling or adult females; annually removing 200 seronegative yearling or adult females; annually harvesting 200 animals from all sex and age classes; and annually vaccinating 200 seronegative yearling or adult females. Goals are to: (1) maintain the composition of the population within the 0.025 and 0.975 quantiles for the stable age distribution of juveniles, adult females, and non-juvenile males in a brucellosis-free, exponentially growing population; (2) maintain the population at levels between 3000 and 3500 individuals; (3) reduce the seroprevalence in the population to $<40 \%$; and (4) reduce the probability of horizontal transmission to half of its value at time 0 .

TABLE 11. Estimates of the number of bison annually removed at the boundary or harvested during hunting seasons.

\begin{tabular}{lcccccr}
\hline \hline & & \multicolumn{2}{c}{ Quantile } & & \\
\cline { 3 - 4 } \multicolumn{1}{c}{ Scenario } & Median & 0.025 & 0.975 & & Minimum & Maximum \\
\hline Remove seropositives & 76 & 13 & 169 & 0 & 299 \\
Remove seronegatives & 58 & 9.5 & 135 & 0 & 258 \\
Hunting & 309 & 52 & 643 & 1 & 1002 \\
Vaccination & 65 & 10 & 154 & 0 & 281 \\
\hline
\end{tabular}

Note: Variation in the estimates arises from annual variation in the number of animals leaving the park. 
TABLE 12. Probability of meeting goals for the Yellowstone bison population and (in parentheses) the proportional change in probability of meeting the goal relative to no action.

\begin{tabular}{|c|c|c|c|c|}
\hline Scenario and goal & Year 0 & Year 1 & Year 3 & Year 5 \\
\hline \multicolumn{5}{|l|}{ No action } \\
\hline Juveniles & 0.99 & $0.5(1)$ & $0.63(1)$ & $0.61(1)$ \\
\hline Males & 0.94 & $0.79(1)$ & 0.77 (1) & 0.74 (1) \\
\hline Females & 0.38 & $0.48(1)$ & $0.43(1)$ & $0.42(1)$ \\
\hline$N>3000$ & 0.94 & $0.96(1)$ & 0.98 (1) & 0.99 (1) \\
\hline$N<3500$ & 0.00 & 0.0018 & $0.0024(1)$ & $0.0014(1)$ \\
\hline Seroprevalence & 0.00 & $0.0062(1)$ & $0.026(1)$ & $0.03(1)$ \\
\hline $\operatorname{Pr}($ transmission $)$ & 0.00 & $0.0026(1)$ & $0.0051(1)$ & $0.0053(1)$ \\
\hline \multicolumn{5}{|l|}{ Remove seropositives } \\
\hline Juveniles & 0.99 & $0.53(1.1)$ & $0.65(1)$ & $0.63(1)$ \\
\hline Males & 0.94 & $0.83(1)$ & $0.81(1.1)$ & $0.78(1.1)$ \\
\hline Females & 0.38 & $0.43(0.9)$ & $0.34(0.8)$ & $0.31(0.75)$ \\
\hline$N>3000$ & 0.94 & $0.92(0.97)$ & $0.95(0.97)$ & $0.97(0.97)$ \\
\hline$N<3500$ & 0.00 & $0.005(2.8)$ & $0.0097(4.1)$ & $0.0088(6.4)$ \\
\hline Seroprevalence & 0.00 & $0.029(4.7)$ & $0.12(4.5)$ & $0.14(4.8)$ \\
\hline $\operatorname{Pr}($ transmission $)$ & 0.00 & $0.0075(2.9)$ & $0.016(3.1)$ & $0.014(2.6)$ \\
\hline \multicolumn{5}{|l|}{ Remove seronegatives } \\
\hline Juveniles & 0.99 & $0.55(1.1)$ & $0.68(1.1)$ & $0.65(1.1)$ \\
\hline Males & 0.94 & $0.83(1)$ & $0.81(1.1)$ & $0.78(1.1)$ \\
\hline Females & 0.38 & $0.43(0.89)$ & $0.34(0.8)$ & $0.32(0.76)$ \\
\hline$N>3000$ & 0.94 & $0.92(0.97)$ & $0.95(0.96)$ & $0.96(0.97)$ \\
\hline$N<3500$ & 0.00 & $0.005(2.8)$ & $0.01(4.3)$ & $0.0098(7.2)$ \\
\hline Seroprevalence & 0.00 & $0.0038(0.61)$ & $0.011(0.42)$ & $0.01(0.34)$ \\
\hline $\operatorname{Pr}($ transmission $)$ & 0.00 & $0.002(0.76)$ & $0.0036(0.72)$ & $0.0049(0.92)$ \\
\hline \multicolumn{5}{|l|}{ Hunting } \\
\hline Juveniles & 0.99 & $0.5(1)$ & $0.63(1)$ & $0.61(1)$ \\
\hline Males & 0.94 & $0.8(1)$ & $0.77(1)$ & $0.74(1)$ \\
\hline Females & 0.38 & $0.48(0.99)$ & $0.42(0.98)$ & $0.41(0.98)$ \\
\hline$N>3000$ & 0.94 & $0.76(0.8)$ & $0.67(0.69)$ & $0.65(0.65)$ \\
\hline$N<3500$ & 0.00 & $0.098(55)$ & $0.19(80)$ & $0.24(170)$ \\
\hline Seroprevalence & 0.00 & $0.006(0.96)$ & $0.026(1)$ & $0.03(1)$ \\
\hline $\operatorname{Pr}($ transmission $)$ & 0.00 & $0.0026(1)$ & $0.005(0.98)$ & $0.0056(1)$ \\
\hline \multicolumn{5}{|l|}{ Vaccination } \\
\hline Juveniles & 0.99 & $0.5(1)$ & $0.63(1)$ & $0.6(1)$ \\
\hline Males & 0.94 & $0.79(1)$ & 0.77 (1) & $0.74(1)$ \\
\hline Females & 0.38 & $0.48(1)$ & $0.43(1)$ & $0.42(1)$ \\
\hline$N>3000$ & 0.94 & $0.96(1)$ & 0.98 (1) & 0.99 (1) \\
\hline$N<3500$ & 0.00 & $0.0016(0.9)$ & $0.0018(0.74)$ & $0.001(0.76)$ \\
\hline Seroprevalence & 0.00 & $0.0057(0.92)$ & $0.025(0.97)$ & $0.034(1.1)$ \\
\hline $\operatorname{Pr}($ transmission $)$ & 0.00 & $0.0025(0.97)$ & $0.0061(1.2)$ & $0.0089(1.7)$ \\
\hline
\end{tabular}

Notes: Estimates include uncertainty in the ability to implement management resulting from stochastic, annual variation in the number of bison that leave the park. The forecast horizon is five years, where year 0 is the last year with data. Management alternatives include: no action, annually removing seropositive yearling or adult females, annually removing seronegative yearling or adult females, annually harvesting animals from all sex and age classes, and annually vaccinating seronegative yearling or adult females. Goals are to: (1) maintain the composition of the population within the 0.025 and 0.975 quantiles for the stable age distribution of juveniles, adult females, and non-juvenile males in a brucellosis-free, exponentially growing population, (2) maintain the population at levels between 3000 and 3500 individuals, (3) reduce the seroprevalence in the population to $<40 \%$, and (4) reduce the probability of horizontal transmission by half of its value at year 0 .

lasts longer than one year in some individuals (characteristic of chronic brucellosis), with other individuals clearing $B$. abortus rapidly after acute infection. We considered alternative model structures that allowed for animals to remain in the infectious stage for adjacent years or return to the infective stage after first moving to the recovered stage. However, parameters of recovery and return to infectiousness were non-identifiable and we instead chose to use the model structure outlined in this paper.
The median of the continuous time rate of frequency dependent transmission $\left(\beta=1.87 \mathrm{yr}^{-1}, \mathrm{BCI}=1.53,2.16\right.$ $\mathrm{yr}^{-1}$; Table 6) is strikingly similar to the point estimate $(\beta \approx 2)$ obtained by Dobson and Meagher (1996). Implicit in the rate of transmission are probabilities that an individual comes in contact with an infectious host and that contact results in infection. Bison investigate birthing events by sniffing and licking birth materials and neonates (Jones et al. 2009), which probably increases the chances of mucosal entry of $B$. abortus. 
Observed contact between bison and birth material increases in males and animals $<2$ years of age (National Park Service, unpublished data). Also, roughly one-half of female bison are exposed to B. abortus by reproductive maturation (Treanor et al. 2011), with log odds of exposure of mature females decreasing with age (National Park Service, unpublished data).

In our model, vertical transmission contributed to increased exposure of juveniles. Uncertainty in this parameter was high (BCI on $v=0.00682,0.289$; Table $6)$. The detailed, process-level studies that formed our prior distribution for the probability of vertical transmission illustrated that nearly as many calves born to seronegative females were seropositive after 6 months of age compared to calves born to seropositive females. Nursing calves can receive maternal $B$. abortus antibodies passed through colostrum that will cause them to react positively on serologic tests. However, this passive immunity typically wanes by the time calves are 6 months of age (Rhyan et al. 2001). If B. abortus transmission to calves was dominated by vertical transmission, we would expect a stronger association of infected newborns ( $>6$ months old) with infected mothers. More plausible, perhaps, is the hypothesis that juvenile and young animals exhibit a higher horizontal rate of transmission compared to adults (Rhyan et al. 2009). However, age-specific parameters for $\beta$ were not identifiable in preliminary models. Further research is needed to explore this hypothesis because increased horizontal transmission rates to younger animals may have noticeable effects on parameter estimates for vertical transmission, recrudescence, and fertility of recovered and infectious bison.

Estimates of median survival probability might appear too low for adult females (Table 6) and high for juveniles. However, 95\% confidence limits on previous estimates of adult survival (Fuller et al. (2007b), 95\% CI =0.87-0.95; Pyne et al. (2010), 95\% $\mathrm{CI}=0.84-0.94)$ overlap the credible intervals of our estimate $\left(\right.$ median $\left.=0.885 \mathrm{yr}^{-1} ; \mathrm{BCI}=0.841,0.93 \mathrm{yr}^{-1}\right)$, suggesting that our estimates are not implausible. The point estimate of juvenile survival (0.76) obtained by Fuller et al. (2007b) was substantially lower than our estimate $($ median $=0.961 ; \mathrm{BCI}=0.837,0.998)$, but the estimate of Pyne et al. (2010) (mean $=0.96, \mathrm{CI}=0.94-$ 98) resembled ours.

The posterior distribution of the growth rate we would expect for the Yellowstone bison population in the absence of infection with brucellosis (median $=1.11$; $\mathrm{BCI}=1.07$, 1.16; Fig. 8A) strongly overlapped the point estimate of the brucellosis-free growth rate of bison populations obtained by Fuller et al. 2007b $(\lambda=1.09)$, Vanvuren and Bray $1986(\lambda=1.10)$, but did not include the estimate of Fuller et al. $(2007 b), \lambda=1.05$. The posterior distribution of the realized growth rate reflecting the influence of Brucellosis (median $\lambda=1.07$; $\mathrm{BCI}=1.03,1.11$; Fig. $8 \mathrm{~B}$ ) was centered on the previous point estimate $(\lambda=1.07)$ for Yellowstone bison obtained by Fuller et al. (2007b). We estimated that brucellosis reduced the growth rate of the Yellowstone bison population by $5 \%$ points (Fig. 8), a seemingly large reduction. However, credible intervals on the difference in population growth rate attributable to the influence of brucellosis ( $\lambda$ (unifected) $-\lambda$ (infected)) slightly overlapped $0(\mathrm{BIC}=-0.014,0.11)$, so we cannot rule out the conclusion that the effects of brucellosis on $\lambda$ are small.

\section{State-space models for understanding diseases}

A central challenge in population ecology is to understand how variation in survival and recruitment governs abundance and demography. Ecologists study populations in two ways: by observing the fates of individuals and by observing the state of the population over time. Both types of data are routinely collected in studies of population dynamics and epidemiology. These data have motivated distinct traditions in disease modeling, the forward approach that predicts unknown states from estimated parameters, and the inverse approach that estimates unknown parameters from observations of states (Hobbs and Ogle 2011, Hobbs and Hooten 2015). Bayesian models, like the one offered here, merge forward and inverse approaches by allowing estimates of parameters derived from studies of individuals to enter the model in prior distributions and by allowing data on the state of the population to enter the model in the likelihoods.

Our work reveals the value of combining multiple types of data in a hierarchical, state-space framework. For example, we made use of prior estimates of recruitment obtained in mark-recapture studies (Fuller et al. 2007b). We also used an extensive time series of observations on the proportion of juveniles in the population and serology of each class. These different data sets taken together allowed us to estimate the probability of horizontal transmission, and hence, the transmission rate $(\beta)$ without direct observations of the transmission process (Fig. 5). This was possible because the number of juveniles in the population was determined by the number of offspring produced per female, and the number of females that were seronegative, seropositive, and seroconverted. The number of females in each infection class, in turn, was determined by the unobserved probability of exposure. Thus, monitoring studies of the demographic composition of the population and process studies estimating vital rates of each infection class combined to inform an unobserved process, brucellosis transmission (Fig. 5).

The success of a research study using Bayesian analysis is often evaluated by comparing the overlap of the posterior distribution of a parameter with its prior distribution to assess how much was learned relative to what was known before the research was done (Gelman et al. 2004, McCarthy 2007). Shrinkage of the posterior relative to the prior or shifts of the centrality of the posterior relative to the prior indicate that the model and data improved parameter estimates relative to what was known before the study. By that standard, our model was 
informative for most parameters of interest (Fig. 6). However, our primary objective was to estimate the values of unknown, latent states and quantities for which there was no prior information, and to enable proper forecasting. Consequently, it does not matter if prior information, or information in the likelihoods, allows these states and quantities to be estimated and predicted. The important outcome is that uncertainty in the priors, or in the likelihoods, influences model estimates of latent states, including the forecasting of future states.

\section{Disease and the conservation of migratory wildlife}

Managing the Yellowstone bison is emblematic of a global challenge in species conservation. A particularly important problem in conserving large mammals worldwide is created by zoonotic diseases transmitted between wildlife, livestock, and people. These diseases isolate conservation areas from the surrounding, humandominated landscape because managers and policy makers often seek to minimize contact between wildlife and the domestic animals that inhabit lands along protected-area boundaries (Newmark 2008). This problem is particularly acute when a disease has been eradicated in domestic livestock because a single transmission from wildlife to livestock can reverse years of expensive effort, harming public health and human livelihoods (Gortázar et al. 2006). As a result, there is often intense political pressure to assure that wildlife hosts are confined to protected areas, confinement that may impede the ability of wildlife to find the habitat needed to meet their seasonally changing requirements. This is the case for many species of ungulates because protected areas often fail to include their complete migratory range. Moreover, when wildlife movements are compressed, crowding of hosts within protected areas can accelerate disease transmission (Lebarbenchon et al. 2006), and in so doing, amplify the risk of transmission to nearby populations of livestock.

Establishing protected areas has been a mainstay of efforts to conserve biological diversity worldwide. This strategy can be successful for sedentary species or species with ranges of movement that are contained within protected-area boundaries. However, the areas of landscapes used by large, migratory mammals often vastly exceed the dimensions of parks and conservation reserves, even the largest ones (Berger 2004, Bolger et al. 2008). There is growing recognition that although protected areas contribute an important component of efforts to conserve the world's biological diversity, they cannot be the sole route to conserving all species (Thirgood et al. 2004, Bolger et al. 2008, Newmark 2008, Craigie et al. 2010), particularly large, migratory species. Instead, effective conservation must work to maintain landscapes that allow migrations to occur (Berger 2004), which requires managing conflict between wildlife and people. Managing the risk of transmission of disease from migratory wildlife to livestock requires a clear understanding of the process of transmission and a way to evaluate alternatives for management. However, even the best, most comprehensive data, hard won by monitoring and research, cannot provide the understanding needed for managing that risk without a framework for modeling and forecasting. We show how a Bayesian state-space model can provide that framework for a species that is iconic for efforts to conserve large mammals in North America.

\section{Conclusions}

The model we describe here is now being used by Yellowstone National Park to support management of brucellosis in bison. Model results were instrumental in informing the decision to forgo implementing a parkwide vaccination program. The low probability of success in reducing brucellosis infection and transmission through vaccination, once uncertainties were considered, was a key factor in this decision (National Park Service and Montana Fish, Wildlife and Parks 2013, National Park Service 2014). A companion state-space model is used to develop annual harvest recommendations and migration forecasts for Yellowstone bison (Geremia et al. $2011,2014 a, b$ ). Using these models to inform adaptive management engages decision makers and stakeholders in an informed conversation, allowing them to consider the consequences of alternative actions with honest assessments of uncertainty.

Estimating process variance separately from observation variance using state-space models is critical to these assessments. Properly assessing process variance, in turn, requires a sustained investment in monitoring the population. Separating process variance from observation variance requires careful sampling design, in particular, replication of observations of population state within years. Short-term, intensive research on population processes such as survival and fecundity cannot substitute for long-term observations because intensive studies cannot characterize the variation in population states that is not accounted for by process models, a characterization that requires quantifying the failures of the model to match observations of population states.

This means that there is no shortcut to supporting adaptive management with state-space models. The Bayesian framework provides a useful way to integrate short-term research on processes with long-term monitoring data. However, these models cannot be built without carefully designed observations of population states accumulated over time.

\section{ACKNOWLEDGMENTS}

N. T. Hobbs received support from the National Park Service (Rocky Mountains Cooperative Ecosystems Studies Unit H120009004), the Yellowstone Park Foundation, the National Science Foundation (Award EF 0914489), and the National Socio-Environmental Synthesis Center (SESYNC), National Science Foundation award DBI-1052875). Paul Cross and Mandan Oli made helpful suggestions on the modeling. Comments by Aaron Ellison, Michael Lavine, Robin Russell, and two anonymous reviewers improved the manuscript. Any 
use of trade, firm, or product names is for descriptive purposes only and does not imply endorsement by the U.S. Government.

\section{Literature Cited}

Adams, L. G. 2002. The pathology of brucellosis reflects the outcome of the battle between the host genome and the brucella genome. Veterinary Microbiology 90:553-561.

Allen, L. J. S., and P. van den Driessche. 2008. The basic reproduction number in some discrete-time epidemic models. Journal of Difference Equations and Applications 14:11271147.

Anderson, R., and R. May. 1979. Population biology of infectious diseases 1. Nature 280:361-367.

Anderson, R., and R. May. 1991. Infectious diseases of humans: dynamics and control. Oxford University Press, Oxford, UK.

Anderson, R. M., and W. Trewhella. 1985. Population dynamics of the badger (Meles meles) and the epidemiology of bovine tuberculosis (Mycobacterium bovis). Philosophical Transactions of the Royal Society B 310:327-381.

Angliss, R. P. 2003. Evaluation of management options for bison and brucellosis in Yellowstone National Park, Wyoming. Dissertation. University of Minnesota, Minneapolis, Minnesota, USA.

Barlow, N. D. 1991. A spatially aggregated disease/host model for bovine $\mathrm{Tb}$ in New Zealand possum populations. Journal of Applied Ecology 28:777-793.

Berger, J. 2004. The last mile: how to sustain long-distance migration in mammals. Conservation Biology 18:320-331.

Berliner, L. M. 1996. Hierarchical Bayesian time-series models. Pages 15-22 in K. Hanson and R. Silver, editors. Maximum entropy and Bayesian methods. Kluwer Academic Publishers, Dordrecht, The Netherlands.

Bidwell, D. 2010. Bison, boundaries, and brucellosis: risk perception and political ecology at Yellowstone. Society and Natural Resources 23:14-30.

Bolger, D. T., W. D. Newmark, T. A. Morrison, and D. F. Doak. 2008. The need for integrative approaches to understand and conserve migratory ungulates. Ecology Letters 11:63-77.

Brooks, S. P., and A. Gelman. 1988. Alternative methods for monitoring convergence of iterative simulations. Journal of Computational and Graphical Statistics 7:434-455.

Brooks, S. P., and A. Gelman. 1997. General methods for monitoring convergence of iterative simulations. Journal of Computational and Graphical Statistics 7:434-455.

Bruggeman, J. E., R. A. Garrott, D. D. Bjornlie, P. J. White, F. G. R. Watson, and J. Borkowski. 2006. Temporal variability in winter travel patterns of Yellowstone bison: the effects of road grooming. Ecological Applications 16:1539-1554.

Cahn, M. L., M. M. Conner, O. J. Schmitz, T. R. Stephenson, J. D. Wehausen, and H. E. Johnson. 2011. Disease, population viability, and recovery of endangered Sierra Nevada bighorn sheep. Journal of Wildlife Management 75:1753-1766.

Caswell, H. 1988. Matrix population models. Sinauer, Sunderland, Massachusetts, USA.

Catlin, J. E., and E. J. Sheehan. 1986. Transmission of bovine brucellosis from dam to offspring. Journal of the American Veterinary Medical Association 188:867-869.

Cheville, N. F., D. R. McCullough, and L. R. Paulson. 1998. Brucellosis in the Greater Yellowstone area. National Academies Press, Washington, D.C., USA.

Clark, J. M. 2007. Models for ecological data. Princeton University Press, Princeton, New Jersey, USA.

Cole, G. F. 1971. An ecological rationale for the natural or artificial regulation of ungulates in parks. Transactions of the North American Wildlife Conference 36:417-425.

Cooch, E., P. B. Conn, S. P. Ellner, A. P. Dobson, and K. H. Pollock. 2012. Disease dynamics in wild populations: modeling and estimation: a review. Journal of Ornithology 152(Supplement 2):S485-S509.

Coyne, M. J., G. Smith, and F. E. McAllister. 1989. Mathematic model for the population biology of rabies in raccoons in the mid-Atlantic states. American Journal of Veterinary Research 50:2148-2154.

Craigie, I. D., J. E. M. Baillie, A. Balmford, C. Carbone, B. Collen, R. E. Green, and J. M. Hutton. 2010. Large mammal population declines in Africa's protected areas. Biological Conservation 143:2221-2228.

Diekman, O., H. Hesterbeak, and T. Britton. 2012. Mathematical tools for understanding infectious disease dynamics. Princeton University Press, Princeton, New Jersey, USA.

Dobson, A., and J. Foufopoulos. 2001. Emerging infectious pathogens of wildlife. Philosophical Transactions of the Royal Society B 356:1001-1012.

Dobson, A., and M. Meagher. 1996. The population dynamics of brucellosis in the Yellowstone National Park. Ecology 77:1026-1036.

Eberhardt, L. L. 2002. A paradigm for population analysis of long-lived vertebrates. Ecology 83:2841-2854.

Ebinger, M., P. Cross, R. Wallen, P. J. White, and J. Treanor. 2011. Simulating sterilization, vaccination, and test-andremove as brucellosis control measures in bison. Ecological Applications, 21:2944-2959.

Ficht, T. A. 2003. Intracellular survival of Brucella: defining the link with persistence. Veterinary Microbiology 92:213-223.

Fuller, J. A., R. A. Garrott, and P. J. White. 2007a. Emigration and density dependence in Yellowstone bison. Journal of Wildlife Management 71:1924-1933.

Fuller, J. A., R. A. Garrott, P. J. White, K. E. Aune, T. J. Roffe, and J. C. Rhyan. 2007b. Reproduction and survival of Yellowstone bison. Journal of Wildlife Management 71:2365-2372.

Gall, D., and K. Nielsen. 2004. Serological diagnosis of bovine brucellosis: a review of test performance and cost comparison. Review of Science and Technology 23:989-1002.

Gall, D., et al. 2000. Validation of the fluorescence polarization assay and comparison to other serological assays for the detection of serum antibodies to Brucella abortus in bison. Journal of Wildlife Diseases 36:469-476.

Gates, C. C., B. Stelfox, T. Muhley, T. Chowns, and R. J. Hudson. 2005. The ecology of bison movements and distribution in and beyond Yellowstone National Park. Technical report. University of Calgary, Alberta, Canada.

Gelman, A., J. B. Carlin, H. S. Stern, and D. B. Rubin. 2004. Bayesian data analysis. Chapman and Hall/CRC, London, UK.

Gelman, A., and J. Hill. 2009. Data analysis using regression and multilievel/hierarchical modeling. Cambridge University Press, Cambridge, UK.

Gelman, A., J. Hwang, and A. Vehtari. 2014. Understanding predictive information criteria for Bayesian models. Statistics and Computing 24(6):997-1016.

Gelman, A., and D. B. Rubin. 1992. Inference from iterative simulation using multiple sequences. Statistical Science 7:457-511.

Geremia, C., R. Wallen, P. J. White, and F. Watson. $2014 a$. Spatial distribution of Yellowstone bison-winter 2015. Technical report. National Park Service, Yellowstone National Park, Mammoth, Wyoming, USA. http://www.ibmp. info/Library/OpsPlans/BisonSpatialDistributions_Final_ Winter2015.pdf

Geremia, C., P. J. White, and R. Wallen. 2014b. Population dynamics and adaptive management of Yellowstone bison. Technical report. National Park Service, Yellowstone National Park, Mammoth, Wyoming, USA. Available at ibmp.info

Geremia, C., P. J. White, R. L. Wallen, F. G. R. Watson, J. J. Treanor, J. Borkowski, C. S. Potter, and R. L. Crabtree. 2011. Predicting bison migration out of Yellowstone National Park using Bayesian models. PLoS ONE 6(2):e16848. 
Gogan, P., K. Podruzny, E. Olexa, H. Pac, and K. Frey. 2005. Yellowstone bison fetal development and phenology of parturition. Journal of Wildlife Management 69:1716-1730.

Gortázar, C., P. Acevedo, F. Ruiz-Fons, and J. Vicente. 2006. Disease risks and overabundance of game species. European Journal of Wildlife Research 52:81-87.

Heidelberger, P., and P. Welch. 1983. Simulation run length control in the presence of an initial transient. Operations Research 31:1109-1044.

Hess, S. C. 2002. Aerial survey methodology for bison population estimation in Yellowstone National Park. Dissertation. Montana State University, Bozeman, Montana, USA.

Hobbs, N. T., and M. B. Hooten. 2015. Bayesian models: a statistical primer for ecologists. Princeton University Press, Princeton, New Jersey, USA.

Hobbs, N. T., and K. Ogle. 2011. Introducing data-model assimilation to students of ecology. Ecological Applications 21:1537-1545.

Hooten, M., and N. T. Hobbs. 2015. A guide to Bayesian model selection for ecologists. Ecological Monographs 85:328.

Huber, J., and P. Nicoletti. 1986. Comparison of the results of card, Rivanol, complement-fixation, and milk ring tests with the isolation rate of Brucella abortus from cattle. American Journal of Veterinary Research 47:1529-1531.

Joly, D., and F. Messier. 2005. The effect of bovine tuberculosis and brucellosis on reproduction and survival of wood bison in Wood Buffalo National Park. Journal of Animal Ecology 74:543-551.

Jones, J., J. Treanor, and R. Wallen. 2009. Parturition in Yellowstone bison. Technical report. Report YCR-2009-01. National Park Service, Mammoth Hot Springs, Wyoming, USA.

Jones, J. D., J. J. Treanor, R. L. Wallen, and P. J. White. 2010. Timing of parturition events in Yellowstone bison (Bison bison): implications for bison conservation and brucellosis transmission risk to cattle. Wildlife Biology 16:333-339.

Keeling, M. J., and P. Rohani. 2008. Modeling infectious diseases in humans and animals. Princeton University Press, Princeton New Jersey, USA.

Kilpatrick, A. M., C. M. Gillin, and P. Daszak. 2009. Wildlifelivestock conflict: the risk of pathogen transmission from bison to cattle outside Yellowstone National Park. Journal of Applied Ecology 46:476-485.

Klepac, P., and H. Caswell. 2011. The stage-structured epidemic: linking disease and demography with a multi-state matrix approach model. Theoretical Ecology 4:301-319.

LaDeau, S. L., G. E. Glass, N. T. Hobbs, A. Latimer, and R. S. Ostfeld. 2011. Data-model fusion to better understand emerging pathogens and improve infectious disease forecasting. Ecological Applications 21:1443-1460.

Lebarbenchon, C., R. Poulin, M. Gauthier-Clerc, and F. Thomas. 2006. Parasitological consequences of overcrowding in protected areas. Ecohealth 3:303-307.

McCarthy, M. A. 2007. Bayesian methods for ecology. Cambridge University Press, Cambridge, UK.

Miller, M. W., N. T. Hobbs, and S. J. Tavener. 2006. Dynamics of prion disease transmission in mule deer. Ecological Applications 16:2208-2214.

Mollison, D. 1995. Epidemic models: their structure and relation to data. Cambridge University Press, Cambridge, UK.

Muths, E., R. D. Scherer, and D. S. Pilliod. 2011. Compensatory effects of recruitment and survival when amphibian populations are perturbed by disease. Journal of Applied Ecology 48:873-879.

National Park Service. 2014. Remote vaccination program to reduce the prevalence of brucellosis in Yellowstone bison. Final environmental impact statement. Technical report. U.S. Department of the Interior, Yellowstone National Park, Mammoth, Wyoming, USA.
National Park Service and Montana Fish, Wildlife and Parks. 2013. Brucellosis science review and workshop. Panelists' report. Technical report. U.S. Department of the Interior, Yellowstone National Park, Mammoth, Wyoming, USA. http://www.nps.gov/yell/learn/nature/upload/Brucellosis Science_RW.pdf

Newmark, W. D. 2008. Isolation of African protected areas. Frontiers in Ecology and the Environment 6:321-328.

Nielsen, K. 2002. Diagnosis of brucellosis by serology. Veterinarian Microbiology 90:447-459.

Nielsen, K., and D. Gall. 2001. Fluorescence polarization assay for the diagnosis of brucellosis: a review. Journal of Immunoassay and Immunochemistry 22:183-201.

Noon, B. R., and J. R. Sauer. 1992. Population models for passerine birds: structure, parameterization, and analysis. Pages 441-464 in D. C. McCullough and R. H. Barrett, editors. Wildlife 2001: populations. Elsevier, London, UK.

Olexa, E. M., and P. J. P. Gogan. 2007. Spatial population structure of Yellowstone bison. Journal of Wildlife Management 71:1531-1538.

Oli, M. K., M. Venkataraman, P. A. Klein, L. D. Wendland, and M. B. Brown. 2006. Population dynamics of infectious diseases: a discrete time model. Ecological Modelling 198:183-194.

Olsen, S., and F. Tatum. 2010. Bovine brucellosis. Veterinary Clinics: Food Animal Practice 26:15-27.

Olsen, S. C. 2010. Brucellosis in the United States: role and significance of wildlife reservoirs. Vaccine 28:F73-F76.

Olsen, S. C., and C. Johnson. 2011. Comparison of abortion and infection after experimental challenge of pregnant bison and cattle with Brucella abortus strain 2308. Clinical and Vaccine Immunology 18:2075-2078.

Perez-Heydrich, C., M. K. Oli, and M. B. Brown. 2012. Population-level influence of a recurring disease on a longlived wildlife host. Oikos 121:377-388.

Peterson, M. J., W. E. Grant, and D. S. Davis. 1991. Bisonbrucellosis management: simulation of alternative strategies. Journal of Wildlife Management 55:205-213.

Philo, L., and W. H. Edwards. 2002. Brucellosis diagnostics. Pages 119-126 in T. J. Kreeger, editor. Brucellosis in elk and bison in the greater Yellowstone area. National Symposium Proceedings. Greater Yellowstone Interagency Brucellosis Committee, Wyoming Game and Fish Department, Cheyenne, Wyoming, USA.

Plumb, G. E., P. J. White, M. B. Coughenour, and R. L. Wallen. 2009. Carrying capacity, migration, and dispersal in Yellowstone bison. Biological Conservation 142:2377-2387.

Plummer, M. 2003. JAGS: a program for analysis of Bayesian graphical models using Gibbs sampling. Proceedings of the Third International Workshop on Distributed Statistical Computing, 20-22 March 2003, Vienna, Austria. http:// www.r-project.org/conferences/DSC-2003/Proceedings/ Plummer.pdf

Plummer, M. 2011a. JAGS: just another Gibbs sampler. Version 3.0.0 user manual. http://sourceforge.net/projects/ mcmc-jags/files/Manuals/3.x/jags_user_manual, pdf

Plummer, M. 2011b. rjags: Bayesian graphical models using MCMC. R package version 3.1.0. http://cran.r-project.org/ package $=$ rjags

Plummer, M., N. Best, K. Cowles, and K. Vines. 2010. coda: output analysis and diagnostics for MCMC. R package version 0.14-4. http://cran.r-project.org/package=coda

Pyne, M. I., K. M. Byrne, K. A. Holfelder, L. McManus, M. Buhnerkempe, N. Burch, E. Childers, S. Hamilton, G. Schroeder, and P. F. Doherty. 2010. Survival and breeding transitions for a reintroduced bison population: a multistate approach. Journal of Wildlife Management 74:1463-1471.

R Core Team. 2012. R: a language and environment for statistical computing. R Foundation for Statistical Computing, Vienna, Austria.

Rhyan, J. C., K. Aune, T. Roffe, D. Ewalt, S. Hennager, T. Gidlewski, S. Olsen, and R. Clarke. 2009. Pathogenesis and 
epidemiology of brucellosis in Yellowstone bison: serologic and culture results from adult females and their progeny. Journal of Wildlife Diseases 45:729-739.

Rhyan, J. C., T. Gidlewski, T. J. Roffe, K. Aune, L. M. Philo, and D. R. Ewalt. 2001. Pathology of brucellosis in bison from Yellowstone National Park. Journal of Wildlife Diseases 37:101-109.

Roffe, T., J. Rhyan, K. Aune, L. Philo, D. Ewalt, T. Gidlewski, and S. Hennager. 1999. Brucellosis in Yellowstone National Park bison: quantitative serology and infection. Journal of Wildlife Management 63:1132-1137.

Rutberg, A. T. 1984. Birth synchrony in American bison (Bison bison): response to predation or season? Journal of Mammalogy 65:418-423.

Samartino, L. E., and F. M. Enright. 1993. Pathogenesis of abortion of bovine brucellosis. Comparative Immunology, Microbiology and Infectious Diseases 16:95-101.

Sikes, R. S., W. L. Gannon, and the Animal Care and Use Committee of the American Society of Mammalogists. 2011. Guidelines of the American Society of Mammalogists for the use of wild mammals in research. Journal of Mammalogy 92:235-253.

Stubben, C. J., and B. G. Milligan. 2007. Estimating and analyzing demographic models using the popbio package in R. Journal of Statistical Software 22:11. http://www.jstatsoft. org/v22/i11

Thirgood, S., A. Mosser, S. Tham, G. Hopcraft, E. Mwangomo, T. Mlengeya, M. Kilewo, J. Fryxell, A. R. E. Sinclair, and M. Borner. 2004. Can parks protect migratory ungulates? The case of the Serengeti wildebeest. Animal Conservation $7: 113-120$.

Thorne, E. T. 2008. Brucellosis. Pages 372-395 in E. S. Williams and I. K. Barker, editors. Infectious diseases of wild mammals. Iowa State University Press, Ames, Iowa, USA.

Treanor, J. J., C. Geremia, P. H. Crowley, J. J. Cox, P. J. White, R. L. Wallen, and D. W. Blanton. 2011. Estimating probabilities of active brucellosis infection in Yellowstone bison through quantitative serology and tissue culture. Journal of Applied Ecology 48:1324-1332.

Treanor, J. J., J. S. Johnson, R. L. Wallen, S. Cilles, P. H. Crowley, J. J. Cox, D. S. Maehr, P. White, and G. E. Plumb. 2010. Vaccination strategies for managing brucellosis in Yellowstone bison. Vaccine 28:F64-F72.

USDI (U.S. Department of the Interior, National Park Service) and USDA (U.S. Department of Agriculture, Forest Service, Animal, and Plant Health Inspection Service). 2000. Record of decision for final environmental impact statement and bison management plan for the state of Montana and Yellowstone National Park. USDI and USDA Technical report, Washington, D.C., USA.

Vanvuren, D., and M. P. Bray. 1986. Population dynamics of bison in the Henry Mountains, Utah. Journal of Mammalogy 67:503-511.

Wallen, R., and D. W. Blanton. 2010. Bison capture and handling protocol for chemical immobilization of bison in Yellowstone National Park. Technical report, Bison Ecology and Management Program. Yellowstone Center for Resources, Mammoth Hot Springs, Wyoming, USA.

Walters, C. J. 1986. Adaptive management of renewable resources. Macmillian, New York, New York, USA.

White, P., R. L. Wallen, C. Geremia, J. J. Treanor, and D. W. Blanton. 2011. Management of Yellowstone bison and brucellosis transmission risk: implications for conservation and restoration. Biological Conservation 144:1322-1334.

White, P. J., and R. L. Wallen. 2012. Yellowstone bisonshould we preserve artificial population substructure or rely on ecological processes? Journal of Heredity 98:1-12.

Yee, S. H., D. L. Santavy, and M. G. Barron. 2011. Assessing the effects of disease and bleaching on Florida Keys corals by fitting population models to data. Ecological Modelling 222:1323-1332.

\section{Supplemental Material}

\section{Ecological Archives}

Appendices A-G and the Supplement are available online: http://dx.doi.org/10.1890/14-1413.1.sm

\section{Data Availability}

Data are available from the Dryad Digital Repository: http://www.dx.doi.org/10.5061/dryad.181qq 